\title{
Detailed Comparison of DNS to PSE for Oblique Breakdown at Mach 3 (Abstract)
}

\author{
Christian S. J. Mayer* and Hermann F. Fasel ${ }^{\dagger}$ \\ The University of Arizona, Tucson, AZ 85721 \\ Meelan Choudhari ${ }^{\ddagger}$ and Chau-Lyan Chang $\ddagger$ \\ NASA Langley Research Center, Hampton, VA 23681
}

\begin{abstract}
A pair of oblique waves at low amplitudes is introduced in a supersonic flat-plate boundary layer. Their downstream development and the concomitant process of laminar to turbulent transition is then investigated numerically using Direct Numerical Simulations (DNS) and Parabolized Stability Equations (PSE). This abstract is the last part of an extensive study of the complete transition process initiated by oblique breakdown at Mach 3. In contrast to the previous simulations, the symmetry condition in the spanwise direction is removed for the simulation presented in this abstract. By removing the symmetry condition, we are able to confirm that the flow is indeed symmetric over the entire computational domain. Asymmetric modes grow in the streamwise direction but reach only small amplitude values at the outflow. Furthermore, this abstract discusses new time-averaged data from our previous simulation CASE 3 and compares PSE data obtained from NASA's LASTRAC code to DNS results.
\end{abstract}

\section{Introduction}

To date, the most dominant nonlinear mechanism that eventually transitions a laminar, supersonic boundary layer to turbulence is still unknown. Knowledge of the relevant nonlinear mechanisms is however mandatory for the accurate determination of transition onset. Previous investigations ${ }^{1-3}$ of the nonlinear transition regime discovered two main nonlinear mechanisms, "oblique breakdown" and "asymmetric subharmonic resonance". Recently, a series of numerical studies ${ }^{4-9}$ using direct numerical simulations (DNS) focused on several unresolved issues related to both mechanisms. In the first part of these studies, ${ }^{4,5}$ the authors were able to identify oblique breakdown in the experiments by Kosinov and his co-workers, ${ }^{2,10-12}$ who investigated asymmetric subharmonic resonance initiated by a wave train in a flat-plate boundary layer at Mach 2 . The identification of oblique breakdown in these experiments is of great importance since a detailed, experimental study of this mechanism has not been performed yet.

The second part of these studies ${ }^{8}$ addressed the question whether oblique breakdown or asymmetric subharmonic resonance, is a stronger nonlinear breakdown mechanism in supersonic boundary layers. In order to answer this question, Mayer et al. ${ }^{8}$ investigated the early nonlinear transition regime initiated by a broad disturbance spectrum on a cone at Mach 3.5. To excite a wide range of disturbance waves, a wave packet was generated by a pulse through a hole on the cone surface. The disturbance spectrum in the frequency-azimuthal mode number plane of the wave packet exhibited traces of oblique breakdown and new features that could be explained with new resonance triads that are composed of three boundary layer modes with three different disturbance frequencies. Furthermore, it was shown that asymmetric subharmonic resonance is a limiting case of these new resonance triads with both secondary waves having subharmonic frequency and that oblique breakdown might be another limiting case with one secondary wave having zero frequency. Moreover, theoretical considerations suggested that oblique breakdown might be a stronger

\footnotetext{
${ }^{*}$ Research Assistant, Dept. of Aerospace \& Mechanical Engineering, Tucson, AZ 85721, AIAA member

${ }^{\dagger}$ Professor, Dept. of Aerospace \& Mechanical Engineering, Tucson, AZ 85721, AIAA member

¥Aerospace Technologist, Computational Aerosciences Branch, Associate Fellow AIAA
} 
nonlinear transition mechanism for two-dimensional boundary layers at supersonic speeds than any other resonance triad.

A nonlinear transition mechanism, however, can only be considered to be an important physical mechanism if it can initiate the entire transition process of a laminar boundary layer to turbulence. This issue was addressed by the final part of the series of numerical studies, ${ }^{6,7,9}$ where the entire transition path of oblique breakdown for a flat-plate boundary layer at Mach 3 was simulated. The DNS in these references, however, had one shortcoming, that is, the flow was assumed to be symmetric in the spanwise direction to reduce computational costs. In order to ensure that this assumption is indeed valid, a new DNS has been conducted in which the artificial symmetry restriction was removed. As a first part, the final conference paper will show results from this simulation and additional longer time-average from the original symmetric simulation of Mayer et al. ${ }^{7}$ (CASE 3). Simulations of the complete transition process as in Mayer et al. ${ }^{7}$ provide a valuable and extensive database that can be utilized for the validation of several different engineering tools across the various stages of transition. The second part of the final conference paper will therefore compare DNS results of the early and late nonlinear stages to data from NASA's LASTRAC code. This code is based on the nonlinear parabolized stability equations.

\section{Simulation Setup for the DNS}

The simulation setup for the DNS in this abstract follows Mayer et al. ${ }^{6,7}$ Supersonic flow at Mach 3 over a flat plate is investigated numerically using spatial DNS. The physical conditions of the simulations match the Princeton wind tunnel conditions: ${ }^{13}$ the unit Reynolds number formed with the free-stream velocity and free-stream viscosity at the inflow was $R e=2.181 \times 10^{6} \mathrm{~m}^{-1}$ and the free-stream temperature is $T_{\infty}^{*}=103.6 \mathrm{~K}$. In the following, details of the computational setup are given and then information on governing equations, boundary conditions, disturbance generation and numerical method are repeated from Mayer et $a l .6,7$ for completeness and convenience of the reader.

\section{A. Computational Setup}

The main parameters for both DNS discussed in this abstract are summarized in table ??. The same nomenclature as in Mayer et al. ${ }^{6,7}$ is employed. Hence, CASE 3 denotes the main case from the older references while CASE 7 is the newest simulation. The overall resolution is exactly the same for both cases. The grid is clustered in the streamwise direction using a fifth-order polynomial and in the wall-normal direction using a third-order polynomial. The computational grid in physical space consists of a total of about 212 million grid points for CASE 3 and 324 million grid points for CASE 7.

Table 1. Main simulation parameters that differ between cases.

\begin{tabular}{|c|c|c|c|}
\hline Parameter & Unit & CASE 3 & CASE 7 \\
\hline \multicolumn{4}{|l|}{ Domain size: } \\
\hline$x_{L}$ & {$[m]$} & 1.145 & 1.050 \\
\hline$y_{H}$ & {$[m]$} & 0.030 & 0.030 \\
\hline \multicolumn{4}{|l|}{ Grid size: } \\
\hline$n_{x}$ & {$[-]$} & 2757 & 2101 \\
\hline$n_{y}$ & {$[-]$} & 301 & 301 \\
\hline$K$ & {$[-]$} & 128 & 256 \\
\hline$n_{z}$ & {$[-]$} & 255 & 512 \\
\hline$n_{x} \times n_{y} \times n_{z}$ & {$[-]$} & $211.6 \mathrm{E} 6$ & $323.8 \mathrm{E} 6$ \\
\hline $\begin{array}{l}\text { Grid resolution (a } \\
\text { points per } \lambda_{x}^{[1,1]} \\
\text { FFT's: }\end{array}$ & $\begin{array}{l}\text { outflow): } \\
{[-]}\end{array}$ & $\sim 440$ & $\sim 440$ \\
\hline Symmetry in $z$ ? & {$[-]$} & yes & no \\
\hline
\end{tabular}

The inflow of the domain for both cases is located at $x_{0}^{*}=0.258 m$ downstream of the leading edge 
of the plate, whereas the outflow ranges from approximately 13.1 (CASE 7) to 14.5 (CASE 3) streamwise wavelengths $\lambda_{x}$ of the oblique fundamental disturbance waves in the linear regime. The domain height is chosen as $y_{H}^{*}=0.030 m \approx 5$ boundary layer thicknesses $\delta$ (laminar) at the outflow, such that even with the large increase in boundary layer thickness caused by the transition process no turbulent flow structures reach the free-stream boundary.

Time-harmonic disturbances with a fundamental frequency of about $f^{*}=6.36 \mathrm{kHz}\left(F=3 \times 10^{-5}\right)$ are introduced through a blowing and suction slot located between $x_{1}^{*}=0.394 m$ and $x_{2}^{*}=0.452 m\left(x_{2}-x_{1} \approx \lambda_{x}\right)$. A discrete wave pair of instability waves with equal but opposite wave angle is excited for all cases. The spanwise wavenumber of $\beta^{*}=211.52 \mathrm{~m}^{-1}$ for the oblique wave pair is chosen such that the generated instability waves experience strong amplification as predicted by LST throughout the entire computational domain. This spanwise wavenumber determines also the domain width of all simulations, i.e. $z_{W}^{*}=\lambda_{z}^{*}=$ $2 \pi / \beta^{*}=0.03 m$.

\section{B. Governing Equations}

The physical problem is governed by the conservation of mass, momentum and total energy with appropriate boundary and initial conditions. The fluid was considered to be a perfect gas with constant specific heat coefficients. The equations were cast in non-dimensional form using an arbitrary reference length (the plate length $L^{*}=0.7239 m$ ) and the free-stream values of the flow quantities at the inflow boundary:

$$
\begin{gathered}
\frac{\partial \rho}{\partial t}+\frac{\partial}{\partial x_{j}}\left(\rho u_{j}\right)=0, \\
\frac{\partial \rho u_{i}}{\partial t}+\frac{\partial}{\partial x_{j}}\left(\rho u_{i} u_{j}+\delta_{i j} p-\tau_{i j}\right)=0, \\
\frac{\partial E_{t}}{\partial t}+\frac{\partial}{\partial x_{j}}\left(\left[E_{t}+p\right] u_{j}-u_{i} \tau_{i j}+q_{j}\right)=0 .
\end{gathered}
$$

The total energy $E_{t}$ and the viscous stress $\tau_{i j}$ are defined as:

$$
E_{t}=\rho\left(\frac{T}{\kappa(\kappa-1) M a^{2}}+\frac{u_{k} u_{k}}{2}\right), \tau_{i j}=\frac{\mu}{R e}\left(\frac{\partial u_{i}}{\partial x_{j}}+\frac{\partial u_{j}}{\partial x_{i}}-\frac{2}{3} \delta_{i j} \frac{\partial u_{k}}{\partial x_{k}}\right) .
$$

The pressure $p$ is computed using the equation of state and the heat flux $q_{i}$ is obtained from Fourier's law:

$$
p=\frac{\rho T}{\kappa M a^{2}}, \quad q_{i}=-\frac{\mu}{(\kappa-1) M a^{2} \operatorname{RePr}} \frac{\partial T}{\partial x_{i}},
$$

with $\kappa=1.4$ and $\operatorname{Pr}=0.71$. The viscosity is calculated using Sutherland's law

$$
\mu(T)=T^{\frac{3}{2}} \frac{1+\frac{C}{T_{\infty}^{*}}}{T+\frac{C}{T_{\infty}^{*}}},
$$

with $C=110.4 K$.

\section{Boundary Conditions}

At the inflow, the conservative quantities $\rho, \rho u_{i}$ and $E_{t}$, obtained from the similarity solution of a compressible flat-plate boundary layer, are specified. The outflow is treated with a buffer domain technique ${ }^{14}$ to avoid reflections of disturbance waves. The buffer domain starts at $x_{3}$ and ends slightly upstream of $x_{L}$ with a length of $x_{L}-x_{3} \sim 0.5 \lambda_{x}$ for all cases. At the free-stream boundary, all total flow quantities are separated into base-flow and disturbance quantities. For the base-flow quantities, a homogeneous von Neumann condition is applied whereas for the disturbance quantities an exponential decay condition is employed that was derived for compressible flow using linear stability considerations. ${ }^{15}$ In the lateral direction, periodicity is assumed. At the wall, the no-slip and no-penetration conditions are used except for the disturbance slot (see below). In addition, for the base flow, the wall temperature is set to the adiabatic wall temperature of the corresponding laminar flow, i.e. the initial condition, whereas temperature fluctuations are assumed to vanish. 


\section{Disturbance Generation}

The flow is forced over the disturbance slot by prescribing a time-harmonic function for the fundamental spanwise Fourier mode of the $v$-velocity. During the startup of the simulation, the forcing amplitude $\tilde{A}(\beta, t)$ is ramped up in time over one disturbance period. The velocity distribution $v_{p}$ over the blowing and suction slot has the shape of a dipole and it is represented by a fifth-order polynomial that is smooth everywhere including at the end points:

$$
v\left(x_{p}, y=0, \beta, t\right)=A(\beta, t) v_{p}\left(x_{p}\right) \cos \left(-\omega t+\theta_{p}(\beta)\right) .
$$

$x_{p}$ was defined as

$$
x_{p}=\frac{2 x-\left(x_{2}+x_{1}\right)}{x_{2}-x_{1}},-1 \leq x_{p} \leq 1
$$

\section{E. Numerical Method}

The governing equations are integrated in time by employing a fourth-order Runge-Kutta scheme. The spatial derivatives are discretized using formally fourth-order split-finite differences in the streamwise and wall-normal directions. ${ }^{16}$ The spanwise direction is assumed to be periodic and therefore transformed into spectral space using Fast Fourier transforms. Moreover, the spanwise discretization is pseudo-spectral, i.e. all nonlinear terms in the governing equations are computed in physical space and then transformed back into spectral space. Two options are implemented for the Fourier Transformations: (i) All flow variables (i.e. $u$-velocity, $v$-velocity, etc.) are assumed to be symmetric to the centerline, except for the spanwise velocity $w$, which is antisymmetric. Symmetric quantities are then transformed into Fourier space using a Fourier cosine transformation and antisymmetric variables $(w)$ are transformed by a Fourier sine transformation. Thus, only one-half spanwise wave length $\lambda_{z}$ has to be computed for this configuration. (ii) No symmetry is assumed and therefore, all variables are transformed using a full Fourier transformation. This option requires the computation of the entire wave length $\lambda_{z}$ in spanwise direction.

The Fourier transformations are based on the VFFTPK library, which can be downloaded from netlib (http://www.netlib.org/vfftpack/). According to this library and its implementation in the Navier-Stokes code, a Fourier cosine transformation into spectral space and its back transformation into physical space are given by

\section{physical $\rightarrow$ spectral:}

$$
\widetilde{\phi}_{k}^{c}=\mathcal{F}(\phi)_{k}^{c} \sim \frac{1}{2\left(n_{z}-1\right)}\left[\phi_{0}^{c}+2 \sum_{l=1}^{n_{z}-1} \phi_{l}^{c} \cos \left(\frac{\pi k l}{n_{z}-1}\right)\right]
$$

spectral $\rightarrow$ physical:

$$
\phi_{l}^{c}=\mathcal{F}^{-1}(\widetilde{\phi})_{l}^{c} \sim \widetilde{\phi}_{0}^{c}+2 \sum_{k=1}^{K-1} \widetilde{\phi}_{k}^{c} \cos \left(\frac{\pi k l}{n_{z}-1}\right)
$$

for $k=0, \ldots, K-1$ and $l=0, \ldots, n_{z}-1$, respectively. $\widetilde{\phi}_{k}^{c}$ represent the Fourier amplitudes for mode $k$. Moreover, $n_{z}$ indicates the number of grid points used for resolving the spanwise direction in physical space over the interval $\left[0,\left(n_{z}-1\right) \Delta z\right]$ with

$$
\Delta z=\frac{\lambda_{z}}{2\left(n_{z}-1\right)}
$$

and $K$ represents the number of modes in Fourier space (for the simulations $n_{z}=2 K-1$ ).

The Fourier sine transformation to spectral space and its back transformation into physical space are as follows

physical $\rightarrow$ spectral:

$$
\widetilde{\phi}_{k}^{s}=\mathcal{F}(\phi)_{k}^{s} \sim-\frac{1}{\left(n_{z}-1\right)} \sum_{l=1}^{n_{z}-1} \phi_{l}^{s} \sin \left(\frac{\pi k l}{n_{z}-1}\right)
$$

spectral $\rightarrow$ physical:

$$
\begin{gathered}
\phi_{l}^{s}=\mathcal{F}^{-1}(\widetilde{\phi})_{l}^{s} \sim-2 \sum_{k=1}^{K-1} \widetilde{\phi}_{k}^{s} \sin \left(\frac{\pi k l}{n_{z}-1}\right) \\
4 \text { of } \square
\end{gathered}
$$


for $k=0, \ldots, K-1$ and $l=0, \ldots ., n_{z}-1$, respectively.

In contrast to a symmetric simulation where only one-half of the spanwise wave length has to be calculated, an asymmetric simulation requires the entire spanwise wave length as computational domain. Hence, for symmetric simulations $n_{z}$ represents the number of grid points in one-half wave length, whereas for asymmetric simulations, this number depicts the grid points in one full spanwise wave length. In this case, the grid spacing in spanwise direction is therefore obtained from

$$
\Delta z=\frac{\lambda_{z}}{\left(n_{z}-1\right)}
$$

The full Fourier transformation for an asymmetric simulation is implemented according to

physical $\rightarrow$ spectral:

$$
\begin{aligned}
\widetilde{\phi}_{0} & \sim \frac{1}{2 n_{z}} \sum_{l=0}^{n_{z}-1} \phi_{l} \\
\widetilde{\phi}_{k}^{c} & \sim \frac{1}{n_{z}} \sum_{l=0}^{n_{z}-1} \phi_{l} \cos \left(\frac{2 \pi k l}{n_{z}}\right) \\
\widetilde{\phi}_{k}^{s} & \sim \frac{1}{n_{z}} \sum_{l=0}^{n_{z}-1} \phi_{l} \sin \left(\frac{2 \pi k l}{n_{z}}\right)
\end{aligned}
$$

spectral $\rightarrow$ physical:

$$
\phi_{l} \sim \widetilde{\phi}_{0}+\sum_{k=1}^{K-1}\left[\widetilde{\phi}_{k}^{c} \cos \left(\frac{2 \pi k l}{n_{z}}\right)+\widetilde{\phi}_{k}^{s} \sin \left(\frac{2 \pi k l}{n_{z}}\right)\right]
$$

with $k=0, \ldots, K-1$ and $l=0, \ldots, n_{z}-1$. As for the symmetric case, $K$ denotes the number of Fourier modes. The entire storage space for the Fourier modes is however $2 K-1$ since the cosine modes and the sine modes have to be stored separately. More details on the numerical method as well as validation cases can be found in the theses of Harris ${ }^{16}$ and von Terzi. ${ }^{17}$

\section{Results and Discussion}

This section discusses some preliminary results. In order to confirm that CASE 3 from Mayer et al. ${ }^{7}$ represents a valuable database for the validation of different engineering tools across the various stages of transition one remaining issue has to be clarified. In CASE 3, the spanwise direction was assumed to be symmetric since so far in the literature, oblique breakdown has always been initiated by two symmetric, oblique, first-mode type instability waves. It is, however, not straight forward to determine if this assumption is indeed justified. Hence, a final simulation where the symmetry restriction is removed is presented in section III Furthermore, this section also shows some results from CASE 3 that are averaged over a longer time interval. The final section (section IIIB) provides a preliminary comparison of PSE results obtained from NASA's LASTRAC code to CASE 3 for the early nonlinear transition regime of oblique breakdown.

\section{A. New DNS results}

The first two figures show results from the new simulation CASE 7. Since there is no symmetry condition in the spanwise direction, the computational domain for CASE 7 is doubled in this direction (see also Table 1 and section IIE). The influence of asymmetric modes on oblique breakdown initiated by two oblique waves with exactly the same amplitude and phase is limited since these modes are only generated by the round-off error of the calculation. In CASE 3 from Mayer et al., ${ }^{7}$ the streamwise position of the final breakup into small-scale structures denotes the location where all modes with frequency unequal to integer multiples of the forcing frequency are strongly amplified. In CASE 7, a similar behavior can be observed. At exactly the same streamwise position (where the breakup into small-scale structures occurs) the asymmetric modes start to be amplified as illustrated by figure \. This figure shows the streamwise velocity disturbance of the first higher Fourier mode in the spanwise direction for the sine and cosine modes. In CASE 3, only cosine 

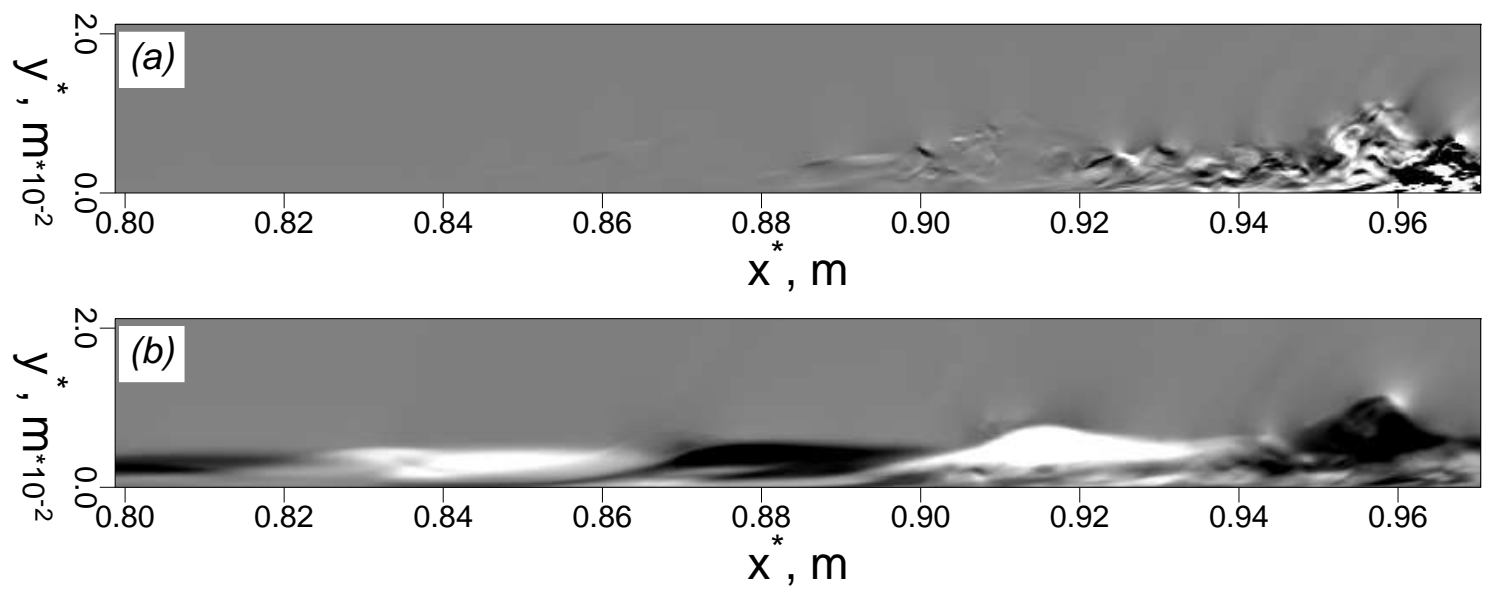

Figure 1. Contours of streamwise velocity $u$ obtained from CASE 7 for the first higher Fourier mode in the spanwise direction: (a) sine mode, (b) cosine mode.

modes were calculated for the streamwise velocity because of symmetry and all sine modes were set to zero. Hence, the amplitude values of the sine mode in figure 1 p provide a measure for the magnitude of asymmetry in CASE 7. Since the sine mode in figure [a is more than 10 orders of magnitude smaller than the cosine mode, CASE 7 remains symmetric even after the breakup into small-scale structures. It is however clearly visible that the asymmetric modes are strongly amplified downstream of this position and will eventually reach high amplitude values in the turbulent region.

The results from figure 1 corroborate that the symmetry assumption in CASE 3 is justified. Thus, for the rest of this section, we concentrate on CASE 3. The time average in Mayer et al. ${ }^{7}$ is calculated over only 12 forcing periods. If this interval length for the time average is sufficient, is depicted in figure 2

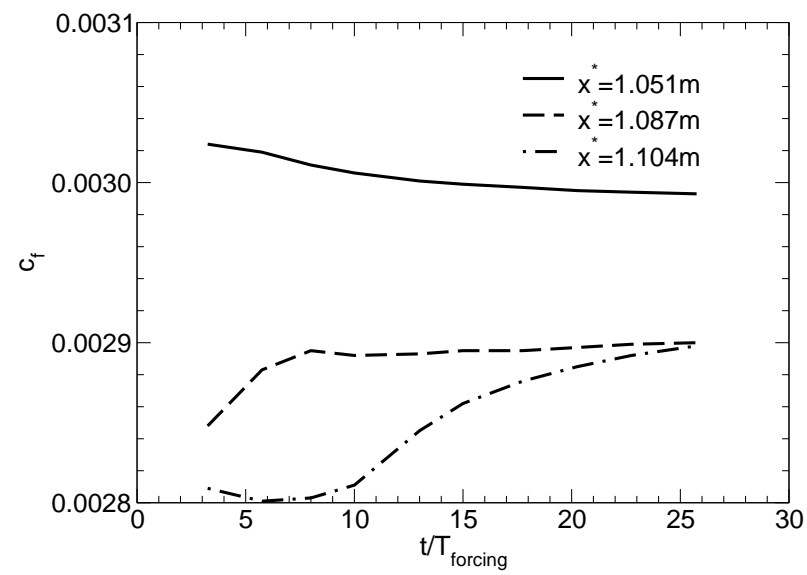

Figure 2. Skin-friction coefficient for CASE 3 as a function of interval length for time-averaging indicated by the number of forcing periods $T_{\text {forcing }}$ at three different streamwise positions.

Figure 2 demonstrates the skin-friction coefficient as a function of the interval length for the time average at three different streamwise positions. For the first two positions, the skin-friction coefficient does not change significantly when the interval length for the time average is increased. At the last position $\left(x^{*}=1.104 m\right)$, however, a longer time average is required. A similar conclusion can be drawn from figure 3 which illustrates the streamwise distribution of selected mean-flow properties from CASE 3 for two different time averages. The curves with 12 forcing periods as time-average interval are very close to the curves with 25.75 forcing periods.

As can be seen in figures 2 and 3 the increase in interval length for the time average does not strongly 

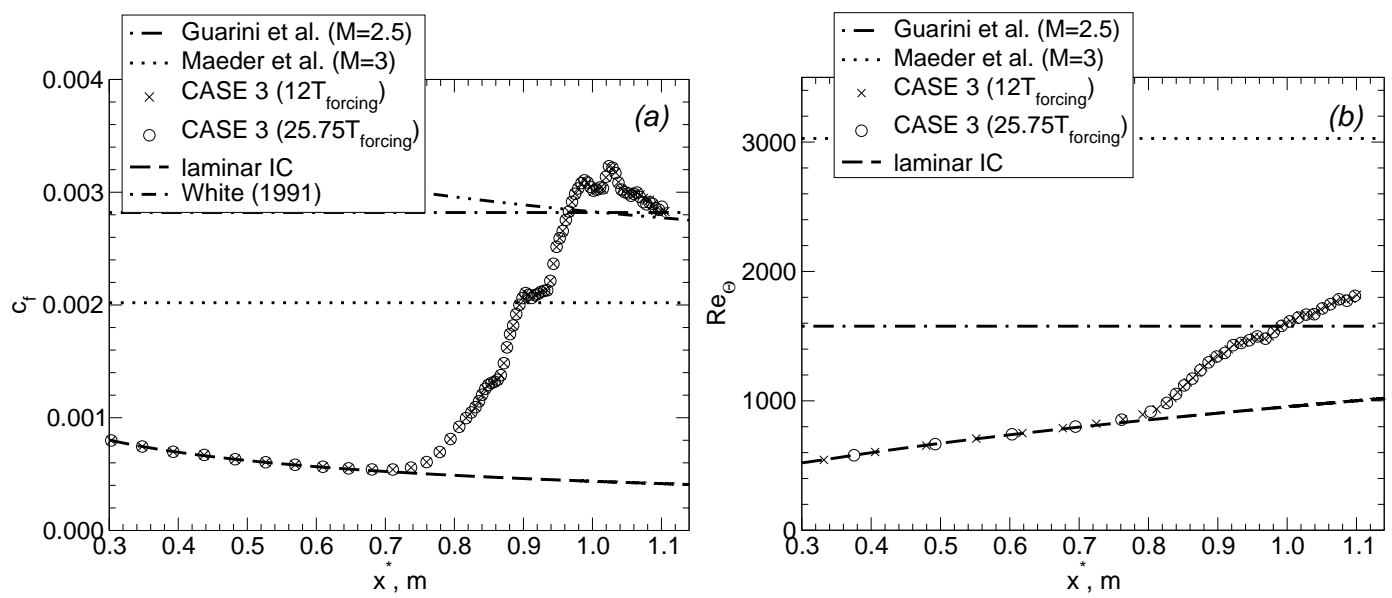

Figure 3. Streamwise development of selected mean-flow properties from CASE 3 in comparison to different values published in the literature for turbulent supersonic flow ${ }^{18,19}$ and theoretical models: ${ }^{20}$ (a) skin-friction coefficient $c_{f}$, (b) Reynolds number based on momentum thickness $\Theta$.

alter the mean values for CASE 3. However, the impact on fluctuation quantities, as for example the r.m.s. values, will be more pronounced. Hence, the final paper will discuss in more detail fluctuation quantities obtained from several different averaging intervals at different streamwise positions. Moreover, the final paper will also give a more detailed discussion of CASE 7.

\section{B. PSE Comparison}

For this abstract, only a preliminary comparison between PSE results and DNS data is provided. Figure 4 shows the streamwise development of wall-normal maximum in streamwise velocity disturbance $u^{\prime}$ for selected Fourier modes. Symbols represent PSE results and lines DNS data. For mode [1,1], the agreement between PSE and DNS is excellent while for the other modes a discrepancy is visible. This discrepancy is due to the different disturbance generation in both methods. Note that the notation $[h, k]$ is used to identify a particular wave according to its frequency $h$ and its spanwise wavenumber $k$. $h$ denotes multiples of the fundamental frequency $f^{*}=6.36 \mathrm{kHz}$ and $k$ multiples of the smallest spanwise wavenumber $\beta^{*}=211.52 \mathrm{~m}^{-1}$. For the final paper, a detailed PSE study will be presented, in which the influence of the forcing method will be addressed.

\section{Conclusions and Future Work}

Transition to turbulence via the oblique breakdown mechanism was investigated for a supersonic flat-plate boundary layer at Mach 3. Our previous studies of the same case focused on the detailed documentation of the different transition stages and demonstrated that oblique breakdown can lead to a fully developed turbulent boundary layer. In these studies, however, the flow was assumed to be symmetric in the spanwise direction. The verification of that assumption was an important part of the results presented in this abstract. To that end, a new simulation without the assumption of spanwise symmetry was performed. The final paper will provide a detailed discussion on this subject. Furthermore, new time-averaged data from CASE 3 of our previous study was shown. In the final paper, the impact of the time-averaging length on fluctuation statistics will be illustrated. Finally, the full paper will provide a detailed comparison of our DNS data to PSE results from NASA's LASTRAC code. 


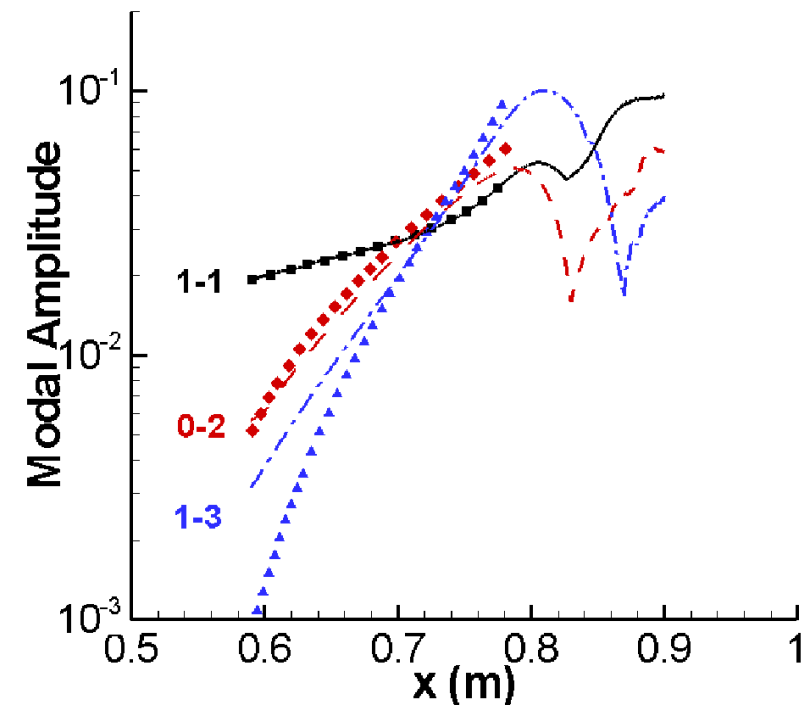

Figure 4. Streamwise development of wall-normal maximum in streamwise velocity disturbance $u^{\prime}$ for selected Fourier modes: lines DNS, symbols PSE.

\section{Acknowledgments}

This work is funded by the Air Force Office for Scientific Research under grant FA9550-08-1-0211 with Dr. John Schmisseur serving as program manager. The computer hours and the technical support provided by NASA Ames are greatly acknowledged.

\section{References}

${ }^{1}$ Fasel, H., Thumm, A., and Bestek, H., "Direct Numerical Simulation of Transition in Supersonic Boundary Layer: Oblique Breakdown," Transitional and Turbulent Compressible Flows, edited by L. D. Kral and T. A. Zang, No. 151 in FED, ASME, 1993, pp. 77-92.

${ }^{2}$ Kosinov, A. D., Semionov, N. V., Shevelkov, S. G., and Zinin, O. I., "Experiments on the Nonlinear Instability of Supersonic Boundary Layers," Nonlinear Instability of Nonparallel Flows, edited by D. T. Valentine, S. P. Lin, and W. R. C. Philips, Springer, 1994, pp. 196-205.

${ }^{3}$ Corke, T. C., Cavalieri, D. A., and Matlis, E., "Boundary-Layer Instability on Sharp Cone at Mach 3.5 with Controlled Input," AIAA J., Vol. 40, No. 5, 2002, pp. 1015-1018.

${ }^{4}$ Mayer, C. S. J., Wernz, S., and Fasel, H. F., "Investigation of Oblique Breakdown in a Supersonic Boundary Layer at Mach 2 Using DNS," AIAA-2007-0949, 2007.

${ }^{5}$ Mayer, C. S. J. and Fasel, H. F., "Investigation of Asymmetric Subharmonic Resonance in a Supersonic Boundary Layer at Mach 2 Using DNS," AIAA-2008-0591, 2008.

${ }^{6}$ Mayer, C. S. J., von Terzi, D. A., and Fasel, H. F., "DNS of Complete Transition to Turbulence Via Oblique Breakdown at Mach 3," AIAA-2008-4398, 2008.

${ }^{7}$ Mayer, C. S. J., von Terzi, D. A., and Fasel, H. F., "DNS of Complete Transition to Turbulence Via Oblique Breakdown at Mach 3: Part. II," AIAA-2009-3558, 2009.

${ }^{8}$ Mayer, C. S. J., Laible, A. C., and Fasel, H. F., "Numerical Investigation of Transition initiated by a Wave Packet on a Cone at Mach 3.5," AIAA-2009-3809, 2009.

${ }^{9}$ von Terzi, D. A., Mayer, C. S. J., and Fasel, H. F., "The Late Nonlinear Stage of Oblique Breakdown to Turbulence in a Supersonic Boundary Layer," Laminar-Turbulent Transition, Springer, 2009, in press.

${ }^{10}$ Kosinov, A. D., Semionov, N. V., and Shevelkov, S. G., "Investigation of Supersonic Boundary Layer Stability and Transition Using Controlled Disturbances," Methods of Aerophysical Research, edited by A. M. Kharitonov, Vol. 2, 1994, pp. $159-166$.

${ }^{11}$ Ermolaev, Y. G., Kosinov, A. D., and Semionov, N. V., "Experimental Investigation of Laminar-Turbulent Transition Process in Supersonic Boundary Layer Using Controlled Disturbances," Nonlinear Instability and Transition in Three-Dimensional Boundary Layers, edited by P. W. Duck and P. Hall, Kluwer Academic Publishers, 1996, pp. 17-26.

${ }^{12}$ Kosinov, A. D., Maslov, A. A., and Semionov, N. V., "An Experimental Study of Generation of Unstable Disturbances on the Leading Edge of a Plate at M=2," J. Appl. Mech. Tech. Phys., Vol. 38, No. 1, 1997, pp. 45-50.

${ }^{13}$ Graziosi, P. and Brown, G. L., "Experiments on Stability and Transition at Mach 3," J. Fluid Mech., Vol. 472, 2002, pp. 83-124. 
${ }^{14}$ Meitz, H. and Fasel, H. F., "A Compact-Difference Scheme for the Navier-Stokes Equations in Vorticity-Velocity Formulation," J. Comp. Phys., Vol. 157, 2000, pp. 371-403.

${ }^{15}$ Thumm, A., Numerische Untersuchungen zum laminar-turbulenten Strömungsumschlag in transsonischen Grenzschichtströmungen, Ph.D. thesis, Universität Stuttgart, 1991.

${ }^{16}$ Harris, P. J., Numerical Investigation of Transitional Compressible Plane Wakes, Ph.D. thesis, The University of Arizona, 1997.

${ }^{17}$ von Terzi, D. A., Numerical Investigation of Transitional and Turbulent Backward-Facing Step Flows, Ph.D. thesis, The University of Arizona, 2004.

${ }^{18}$ Guarini, S. E., Moser, R. D., Shariff, K., and Wray, A., "Direct Numerical Simulation of a Supersonic Turbulent Boundary Layer at Mach 2.5," J. Fluid Mech., Vol. 414, 2000, pp. 1-33.

${ }^{19}$ Maeder, T., Adams, N. A., and Kleiser, L., "Direct simulations of turbulent supersonic boundary layers by an extended temporal approach," J. Fluid Mech., Vol. 429, 2001, pp. 187-216.

${ }^{20}$ White, F. M., Viscous Fluid Flow, McGraw-Hill, 1991. 


\title{
Detailed Comparison of DNS to PSE for Oblique Breakdown at Mach 3
}

\author{
Christian S. J. Mayer* and Hermann F. Fasel ${ }^{\dagger}$ \\ The University of Arizona, Tucson, AZ 85721 \\ Meelan Choudhari ${ }^{\ddagger}$ and Chau-Lyan Chang $\ddagger$ \\ NASA Langley Research Center, Hampton, VA 23681
}

\begin{abstract}
Oblique breakdown in a supersonic flat-plate boundary layer is investigated using Direct Numerical Simulations (DNS) and Parabolized Stability Equations (PSE). This paper constitutes an extension to our previous studies of the complete transition regime of oblique breakdown. In these studies, the flow was assumed to be symmetric in the spanwise direction. A new DNS has been performed where the symmetry condition was removed. This simulation demonstrates that the "classical" oblique breakdown mechanism initialized by two symmetric instability waves with equal disturbance amplitudes loses its symmetry late in the turbulent stage for a low-noise environment. Hence, for the streamwise extent of the computational domain in our studies, the symmetry condition is justified. Furthermore, new data from a longer time average of the original symmetric simulation of oblique breakdown (CASE 3) are discussed. These data verify that a converged time average is reached. The final part of the paper focuses on a comparison of PSE results obtained from NASA's LASTRAC code to the DNS results. This comparison corroborates that the nonlinear PSE approach can successfully predict transition onset and that despite the large amplitude forcing used to introduce the oblique mode disturbances in the DNS, the latter constitutes a generic reference case for oblique breakdown at Mach 3 and, therefore, can be used to validate reduced order models for the full transition zone.
\end{abstract}

\section{Nomenclature}

\begin{tabular}{llll} 
Latin & & \multicolumn{3}{l}{ Greek } \\
$A$ & Disturbance amplitude, & $-\alpha_{i}$ & Streamw. amplification rate, \\
$c_{f}$ & Skin friction coefficient, & $\alpha_{r}, \beta$ & Streamw., spanw. wave number, \\
$c_{p}$ & Specific heat at const. pressure & $\delta$ & Boundary layer thickness, \\
$E_{t}$ & Total energy, & $\delta_{i j}$ & Kronecker delta, \\
$f$ & Frequency, & $\Delta t$ & Interval length for time average, \\
$F$ & Normalized frequency, & $\Delta z$ & Grid spacing in $z$, \\
$i$ & $\sqrt{(-1)}$, & $\theta$ & Phase in streamwise direction, \\
$k$ & Thermal conductivity, & $\Theta$ & Momentum thickness, \\
$K$ & Spanwise resolution (spectral), & $\gamma$ & Ratio of specific heats, \\
$L$ & Reference length, & $\lambda$ & Wavelength, \\
$M$ & Mach number, & $\mu$ & Dynamic viscosity, \\
$n_{x}, n_{y}, n_{z}$ & Number of points in $x, y, z$, & $\nu$ & Kinematic viscosity, \\
$n_{t}$ & Number of saved timesteps, & $\rho$ & Density, \\
$p$ & Pressure, & $\tau_{i j}$ & Stress tensor,
\end{tabular}

* present affiliation: ExxonMobil Upstream Research Company, Houston, TX 77252, AIAA member

${ }^{\dagger}$ Professor, Dept. of Aerospace \& Mechanical Engineering, Tucson, AZ 85721, AIAA member

$\ddagger$ Aerospace Technologist, Computational AeroSciences Branch, Associate Fellow AIAA 


\begin{tabular}{|c|c|c|c|}
\hline $\operatorname{Pr}$ & Prandtl number, & $\phi$ & A flow quantity, \\
\hline$q_{i}$ & Heat flux vector, & $\omega$ & Angular frequency, \\
\hline$Q$ & $Q$-criterion, & & \\
\hline$R e$ & Reynolds number based on $L$, & \multicolumn{2}{|c|}{ Superscripts } \\
\hline$R e_{\Theta}$ & Reynolds number based on $\Theta$, & $*$ & Dimensional value, \\
\hline$R_{x}$ & Local Reynolds number, & ' & Disturbance value, \\
\hline$u_{i}$ & Velocity vector, & - & Time-averaged quantity, \\
\hline$t$ & Time, & $s$ & Anti-symmetric (sine mode), \\
\hline$T$ & Temperature, & $c$ & Symmetric (cosine mode), \\
\hline$T_{\text {forcing }}$ & Forcing period, & $\sim$ & In Fourier space, \\
\hline$x_{i}$ & Coordinate vector, & & \\
\hline$x, y, z$ & Streamw., wall-normal, spanw. directions, & \multicolumn{2}{|c|}{ Subscripts } \\
\hline$x_{0}, x_{L}$ & Location of inflow, outflow, & {$[h, k]$} & Modes in [time, spanw. direction] \\
\hline$x_{1}, x_{2}$ & Start, end of disturbance hole, & $\infty$ & Free-stream value, \\
\hline$x_{3}$ & Start of buffer domain, & $w$ & Wall value. \\
\hline$y_{H}$ & Domain height, & & \\
\hline$z_{W}$ & Domain width, & & \\
\hline
\end{tabular}

\section{Introduction}

Boundary layer transition has important aerodynamic design implications on supersonic and hypersonic vehicles due to the strong increase in aerothermal loads. During the design process of the Space Shuttle Orbiter, boundary layer transition was recognized to be a significant aerothermodynamic challenge. ${ }^{1}$ For accurate and successful prediction of transition onset, the transition process must be better understood in order to provide the future design community reliable physical prediction models. ${ }^{2}$ These physical models need to incorporate the main transition stages, namely the receptivity regime, the initial linear disturbance development and the early nonlinear regime leading to the final breakdown.

While the theoretical models governing the receptivity regime and the linear disturbance development are well established, ${ }^{3-5}$ the nonlinear regime has received less attention. The knowledge of the relevant nonlinear mechanisms is, however, mandatory for the accurate determination of transition onset. Previous investigations $^{6-8}$ of the nonlinear transition regime for a two-dimensional, supersonic boundary layer discovered two main nonlinear mechanisms, oblique breakdown and asymmetric subharmonic resonance. Recently, a series of numerical studies ${ }^{9-14}$ using direct numerical simulations (DNS) focused on several unresolved issues related to both mechanisms. In the first part of these studies, ${ }^{9,10}$ the authors were able to identify oblique breakdown in the experiments by Kosinov and co-workers, ${ }^{7,15-17}$ who investigated asymmetric subharmonic resonance initiated by a wave train in a flat-plate boundary layer at Mach 2 . The discovery of oblique breakdown in these experiments is of great importance since a detailed, experimental study of this mechanism has not been performed yet.

The second part of these studies ${ }^{13}$ addressed the question whether oblique breakdown or asymmetric subharmonic resonance is a stronger nonlinear breakdown mechanism in supersonic boundary layers. In order to answer this question, Mayer et al. ${ }^{13}$ investigated the early nonlinear transition regime initiated by a broad disturbance spectrum on a cone at Mach 3.5. To excite a wide range of disturbance waves, a wave packet was generated by a pulse through a hole on the cone surface. The disturbance spectrum in the frequency-azimuthal mode number plane of the wave packet exhibited traces of oblique breakdown and new features that could be explained with new resonant triads that are composed of three boundary layer modes with three different disturbance frequencies. Furthermore, it was shown that asymmetric subharmonic resonance can be understood as a limiting case of these new resonance triads with both secondary waves having subharmonic frequency and that oblique breakdown might be another limiting case with one secondary wave having zero frequency. Moreover, theoretical considerations suggested that oblique breakdown might be a stronger nonlinear transition mechanism for two-dimensional boundary layers at supersonic speeds than any other resonance triad. A nonlinear transition mechanism, however, can only be considered to be an important physical mechanism if it can initiate the entire transition process of a laminar boundary layer to turbulence. This issue was addressed by the final part of the series of numerical studies, ${ }^{11,12,14}$ where the 
entire transition path of oblique breakdown for a flat-plate boundary layer at Mach 3 was simulated. Similar computations for first-mode type waves in a Mach 4.5 boundary layer were reported earlier by Jiang et al. ${ }^{18}$

The present paper complements the study by Mayer et al. ${ }^{11,12,14}$ via one additional DNS computation. In the earlier investigations, Mayer et al. ${ }^{11,12,14}$ assumed symmetry with respect to the spanwise direction to reduce computational costs. In order to ensure that this assumption is indeed valid, a new DNS has been conducted in which the artificial symmetry restriction was removed. Furthermore, the original symmetric simulation of Mayer et al. ${ }^{11,12,14}$ was continued and additional time-dependent data were saved in order to extend the interval for the time average and, hence, to ensure the convergence of statistical quantities.

A major theme of this paper focuses on a detailed comparison between results obtained from a parabolized stability equation (PSE) approach using the Langley Stability and Transition Analysis Codes (LASTRAC $)^{19,20}$ and the DNS results. DNS results of the complete transition process as discussed in this paper, together with Mayer et al. ${ }^{11,12}$ and von Terzi et al. ${ }^{14}$ constitute a valuable and extensive database that can be utilized for the validation of several different engineering tools across the various stages of transition. With the development of LASTRAC, NASA Langley Research Center aims to create an integrated tool kit that can be employed for conventional N-factor calculations (based on LST or PSE) and more sophisticated simulations from the receptivity process to the early nonlinear transition stages. Thus, a successful comparison between LASTRAC and the DNS results would validate LASTRAC's nonlinear prediction capabilities.

\section{Governing Equations}

Supersonic flow at Mach 3 over a flat plate is investigated numerically using spatial DNS, linear, and nonlinear PSE. The governing equations are derived for a rectangular coordinate system with $x$ as streamwise, $y$ as wall-normal, and $z$ as spanwise coordinates. The physical conditions of the simulations match the Princeton wind tunnel conditions. ${ }^{21}$ The unit Reynolds number based on the free-stream velocity and free-stream viscosity is $R e=2.181 \times 10^{6} \mathrm{~m}^{-1}$ and the free-stream temperature is $T_{\infty}^{*}=103.6 \mathrm{~K}$. Note that $*$ indicates dimensional values. The fluid is considered to be a perfect gas with constant specific heat coefficients. The flow quantities are nondimensionalized by their approach-flow values, indicated by the subscript $\infty$, except for the pressure and the total energy, which are scaled by the dynamic pressure $\rho_{\infty}^{*} U_{\infty}^{*}{ }^{2}$. The flow evolution is governed by the conservation of mass, momentum, and total energy:

$$
\begin{gathered}
\frac{\partial \rho}{\partial t}+\frac{\partial}{\partial x_{j}}\left(\rho u_{j}\right)=0, \\
\frac{\partial \rho u_{i}}{\partial t}+\frac{\partial}{\partial x_{j}}\left(\rho u_{i} u_{j}+\delta_{i j} p-\tau_{i j}\right)=0, \\
\frac{\partial E_{t}}{\partial t}+\frac{\partial}{\partial x_{j}}\left(\left[E_{t}+p\right] u_{j}-u_{i} \tau_{i j}+q_{j}\right)=0,
\end{gathered}
$$

where the symbols $\rho$ and $u_{i}$ denote the fluid density and the velocity vector, respectively.

The total energy $E_{t}$ and the viscous stress $\tau_{i j}$ are defined as:

$$
E_{t}=\rho\left(\frac{T}{\gamma(\gamma-1) M^{2}}+\frac{u_{k} u_{k}}{2}\right), \tau_{i j}=\frac{\mu}{R e}\left(\frac{\partial u_{i}}{\partial x_{j}}+\frac{\partial u_{j}}{\partial x_{i}}-\frac{2}{3} \delta_{i j} \frac{\partial u_{k}}{\partial x_{k}}\right),
$$

with $T$ as temperature. The pressure $p$ is computed using the equation of state and the heat flux $q_{i}$ is obtained from Fourier's law:

$$
p=\frac{\rho T}{\gamma M^{2}}, \quad q_{i}=-\frac{\mu}{(\gamma-1) M^{2} \operatorname{RePr}} \frac{\partial T}{\partial x_{i}} .
$$

The viscosity is calculated using Sutherland's law

$$
\mu(T)=T^{\frac{3}{2}} \frac{1+\frac{C}{T_{\infty}^{*}}}{T+\frac{C}{T_{\infty}^{*}}},
$$

with $C=110.4 K$. Bulk viscosity is negligible. 
The nondimensionalization of the governing equations introduces the Mach number $M$, the Prandtl number $P r$, the ratio of specific heats, and the Reynolds number Re. The first three nondimensional parameters are defined as

$$
M=\frac{U_{\infty}^{*}}{a_{\infty}^{*}}=\frac{U_{\infty}^{*}}{\sqrt{(\gamma-1) c_{p \infty}^{*} T_{\infty}^{*}}}=3, \quad \operatorname{Pr}=\frac{\mu^{*} c_{p \infty}^{*}}{k^{*}}=0.71, \quad \text { and } \quad \gamma=1.4 .
$$

with $c_{p \infty}^{*}, k^{*}$, and $a_{\infty}^{*}$ being the specific heat at constant pressure, the thermal conductivity, and the speed of sound of the approach flow, respectively. The Reynolds number,

$$
R e=\frac{\rho_{\infty}^{*} U_{\infty}^{*} L^{*}}{\mu_{\infty}^{*}}
$$

is based on a reference length $L^{*}$, which is a constant for both numerical approaches. For the DNS, the plate length in the experiment is used while for the PSE, the similarity boundary-layer length scale $\left(L^{*}=\sqrt{\frac{\nu_{\infty}^{*} x^{*}}{U_{\infty}^{*}}}\right)$ at the initial location $x_{0}^{*}$ is chosen,

$$
L_{D N S}^{*}=0.7239 \quad \text { and } \quad L_{P S E}^{*}=\sqrt{\frac{\nu_{\infty}^{*} x_{0}^{*}}{U_{\infty}^{*}}} .
$$

\section{A. Modifications for the PSE Approach}

Eqs. (1) to (6) are solved directly in the DNS. For the PSE approach, the governing equations are further simplified as discussed in detail by Chang. ${ }^{19}$ All flow quantities $\phi$ are decomposed into the laminar mean flow solution $\Phi$ and a disturbance fluctuation $\phi^{\prime}$

$$
\phi=\Phi+\phi^{\prime} .
$$

The disturbance fluctuation can be represented by the following wave ansatz

$$
\phi^{\prime}=\epsilon \tilde{\phi}(x, y) \exp \left[i\left(\int_{x_{0}}^{x} \alpha(\xi) d \xi+\beta z-\omega t\right)\right],
$$

with $\alpha=\alpha_{r}+i \alpha_{i}$ as complex streamwise wave number, $\beta$ as spanwise wave number, and $\omega$ as angular frequency. The integral form of the streamwise wave number is used to allow for a streamwise variation of $\alpha$ and to record the history effect. The shape function $\tilde{\phi}$ is not only dependent on the wall-normal direction $y$ as for linear stability theory (LST), but also on the streamwise coordinate $x$. Thus, non-parallel effects can be captured in the PSE approach through the variation of the streamwise wave number and the shape function. Substituting Eqs. (10) and (11) into Eqs. (10) to (6) and subtracting the governing equations for the mean flow, leads to the final equation for the PSE approach

$$
L \tilde{\phi}=\tilde{f}, \quad \text { with } \quad L=\tilde{A} \partial_{x}+\tilde{B} \partial_{y}+\tilde{D}-U_{y y} \partial_{y y} .
$$

Here, the diffusion terms with respect to the streamwise direction $x$ are neglected. The forcing function $\tilde{f}$ contains the Fourier transform of the forcing applied in the PSE analysis and also the nonlinear terms for the nonlinear PSE approach. The coefficient matrices $\tilde{A}, \tilde{B}$, and $\tilde{C}$ are functions of all wave numbers and the mean flow and can be found in Chang. ${ }^{19}$

For completeness, the frequency $\omega$ in Eq. (11) is usually rescaled in stability calculations according to

$$
F=\frac{\omega}{R_{x}}
$$

where the Reynolds number $R_{x}$ is based on the similarity boundary-layer length scale $L^{*}=\sqrt{\frac{\nu_{\infty}^{*} x^{*}}{U_{\infty}^{*}}}$,

$$
R_{x}=\sqrt{\frac{U_{\infty}^{*} x^{*}}{\nu_{\infty}^{*}}}
$$




\section{Simulation Setup for the DNS}

The simulation setup for the DNS follows Mayer et al. ${ }^{11,12}$ and is repeated here for the convenience of the reader. In the next sections, the computational setup, the numerical method, boundary conditions and the disturbance generation is introduced. The purpose of the detailed description of the simulation setup, and especially the focus on the spanwise Fourier transformation in the numerical method section, is to provide the reader with all information necessary for the repetition of the simulations. Note that there is also a note on post-processing that discusses the temporal Fourier transformations applied to the DNS data to obtain the spectral composition of the disturbance field.

\section{A. Computational Setup}

The main parameters for both DNS discussed in this paper are summarized in Table 1 The same nomenclature as in Mayer et al. ${ }^{11,12}$ is employed (see also nomenclature). Hence, CASE 3 denotes the main case from the older references while CASE 7 is the new asymmetric simulation. The streamwise and wall-normal resolution is exactly the same for both cases. The grid is clustered in the streamwise direction using a fifth-order polynomial and in the wall-normal direction using a third-order polynomial. The computational grid in physical space consists of a total of about 212 million grid points for CASE 3 and 324 million grid points for CASE 7 . The difference in the overall number of grid points between both cases is mainly due to the slightly shorter domain in streamwise direction and the larger domain in spanwise direction for CASE 7.

Table 1. Grid and simulation characteristics. Note that the notation $[h, k]$ is used to identify a particular wave according to its frequency $h$ and its spanwise wave number $k$. $h$ denotes multiples of the fundamental frequency and $k$ represents multiples of the smallest spanwise wave number.

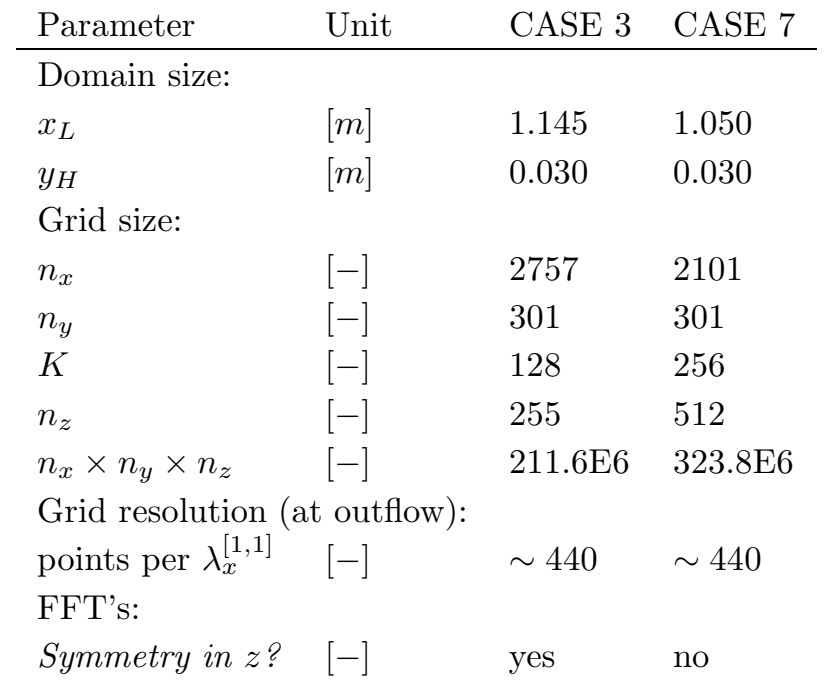

The computational setup is illustrated in Fig. 10 The inflow of the domain for both cases is located at $x_{0}^{*} \simeq 0.258 \mathrm{~m}$ downstream of the leading edge of the plate, whereas the outflow ranges from approximately 13.1 (CASE 7) to 14.5 (CASE 3) streamwise wavelengths $\lambda_{x}$ of the oblique fundamental disturbance waves in the linear regime measured from the inflow. The domain height is chosen as $y_{H}^{*} \simeq 0.030 m \approx 5$ boundary layer thicknesses $\delta$ (laminar) at the outflow, such that even with the high increase in boundary layer thickness caused by the transition process no turbulent flow structures reach the free-stream boundary.

Time-harmonic disturbances with a fundamental frequency of about $f^{*}=6.36 \mathrm{kHz}\left(F=3 \times 10^{-5}\right)$ are introduced through a blowing and suction slot located between $x_{1}^{*} \simeq 0.394 m$ and $x_{2}^{*} \simeq 0.452 m\left(x_{2}-x_{1} \approx \lambda_{x}\right)$. A pair of instability waves with equal but opposite wave angle is excited via the slot. The spanwise wave number of $\beta^{*} \simeq 211.52 \mathrm{~m}^{-1}$ for the oblique wave pair is chosen such that the generated instability waves experience strong amplification as predicted by LST throughout the entire computational domain. This spanwise wave number also determines the corresponding domain width for the simulations, i.e. $z_{W}^{*}=\lambda_{z}^{*}=$ 
$2 \pi / \beta^{*} \simeq 0.03 m$. For CASE 3 , only half of this spanwise domain width is simulated because of symmetry, while, for CASE 7, the entire domain must be considered.

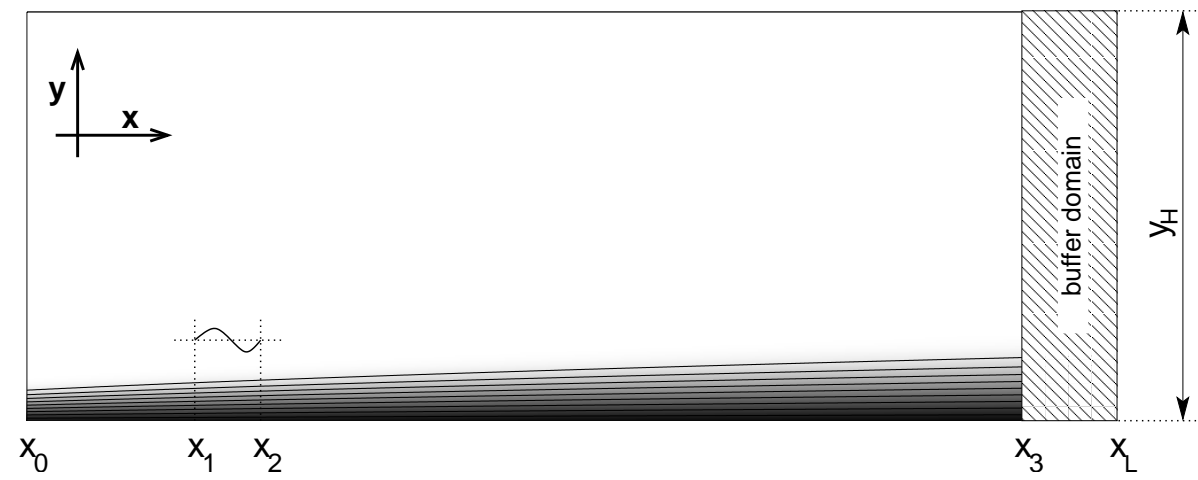

Figure 1. Illustration of the computational domain for CASE 3 and CASE 7.

\section{B. Numerical Method}

The governing equations are integrated in time by employing a fourth-order Runge-Kutta scheme. The spatial derivatives are discretized using formally fourth-order split-finite differences in the streamwise and wallnormal directions. The spanwise direction is assumed to be periodic and, therefore, transformed into spectral space using Fast Fourier Transforms (FFT). Moreover, the spanwise discretization is pseudo-spectral, ${ }^{22}$ i.e. all nonlinear terms in the governing equations are computed in physical space and then transformed back into spectral space. Two options are available for the spanwise Fourier transformations: (i) All flow variables (i.e. streamwise velocity $u$, wall-normal velocity $v$, etc.) are assumed to be symmetric to the centerplane, except for the spanwise velocity $w$, which is antisymmetric. Symmetric quantities are then transformed into Fourier space using a Fourier cosine transformation and antisymmetric variables $(w)$ are transformed by a Fourier sine transformation. Thus, only one-half spanwise wave length $\lambda_{z}$ has to be computed for this configuration. (ii) No symmetry is assumed and, therefore, all variables are transformed using a full Fourier transformation. This option requires the computation of the entire spanwise wave length $\lambda_{z}$.

The Fourier transformations are based on the VFFTPK library, which can be downloaded from netlib (http://www.netlib.org/vfftpack/). According to this library and its implementation in the Navier-Stokes code, a Fourier cosine transformation into spectral space and its inverse transformation into physical space are given by

physical $\rightarrow$ spectral:

$$
\widetilde{\phi}_{k}^{c} \sim \frac{1}{2\left(n_{z}-1\right)}\left[\phi_{0}^{c}+2 \sum_{l=1}^{n_{z}-1} \phi_{l}^{c} \cos \left(\frac{\pi k l}{n_{z}-1}\right)\right],
$$

spectral $\rightarrow$ physical:

$$
\phi_{l}^{c} \sim \widetilde{\phi}_{0}^{c}+2 \sum_{k=1}^{K-1} \widetilde{\phi}_{k}^{c} \cos \left(\frac{\pi k l}{n_{z}-1}\right),
$$

for $k=0, \ldots, K-1$ and $l=0, \ldots, n_{z}-1$, respectively. $\widetilde{\phi}_{k}^{c}$ represents the Fourier amplitude for mode $k$. Moreover, $n_{z}$ indicates the number of grid points used for resolving the spanwise direction in physical space over the interval $\left[0,\left(n_{z}-1\right) \Delta z\right]$ with

$$
\Delta z=\frac{\lambda_{z}}{2\left(n_{z}-1\right)}
$$

and $K$ represents the number of modes in Fourier space (for the simulations $n_{z}=2 K-1$ ).

The Fourier sine transformation to spectral space, with $\widetilde{\phi}_{k}^{s}$ as the Fourier amplitude, and its inverse into 
physical space are

$$
\begin{aligned}
& \text { physical } \rightarrow \text { spectral: } \\
& \qquad \widetilde{\phi}_{k}^{s} \sim-\frac{1}{\left(n_{z}-1\right)} \sum_{l=1}^{n_{z}-1} \phi_{l}^{s} \sin \left(\frac{\pi k l}{n_{z}-1}\right),
\end{aligned}
$$

spectral $\rightarrow$ physical:

$$
\phi_{l}^{s} \sim-2 \sum_{k=1}^{K-1} \widetilde{\phi}_{k}^{s} \sin \left(\frac{\pi k l}{n_{z}-1}\right),
$$

for $k=0, \ldots, K-1$ and $l=0, \ldots, n_{z}-1$, respectively.

In contrast to a symmetric simulation where only one-half of the spanwise wave length has to be calculated, an asymmetric simulation requires the entire spanwise wave length as computational domain. Hence, for symmetric simulations $n_{z}$ represents the number of grid points in one-half wave length, whereas for asymmetric simulations, this number depicts the grid points in one full spanwise wave length. In this case, the grid spacing in the spanwise direction is therefore obtained from

$$
\Delta z=\frac{\lambda_{z}}{\left(n_{z}-1\right)} .
$$

The full Fourier transformations for an asymmetric simulation are

$$
\text { physical } \rightarrow \text { spectral: }
$$

$$
\begin{aligned}
\widetilde{\phi}_{0} & \sim \frac{1}{2 n_{z}} \sum_{l=0}^{n_{z}-1} \phi_{l}, \\
\widetilde{\phi}_{k}^{c} & \sim \frac{1}{n_{z}} \sum_{l=0}^{n_{z}-1} \phi_{l} \cos \left(\frac{2 \pi k l}{n_{z}}\right), \\
\widetilde{\phi}_{k}^{s} & \sim \frac{1}{n_{z}} \sum_{l=0}^{n_{z}-1} \phi_{l} \sin \left(\frac{2 \pi k l}{n_{z}}\right),
\end{aligned}
$$

spectral $\rightarrow$ physical:

$$
\phi_{l} \sim \widetilde{\phi}_{0}+\sum_{k=1}^{K-1}\left[\widetilde{\phi}_{k}^{c} \cos \left(\frac{2 \pi k l}{n_{z}}\right)+\widetilde{\phi}_{k}^{s} \sin \left(\frac{2 \pi k l}{n_{z}}\right)\right],
$$

with $k=0, \ldots, K-1$ and $l=0, \ldots, n_{z}-1$. As for the symmetric case, $K$ denotes the number of Fourier modes. The entire storage space scales by $2 K-1$ since the cosine modes and the sine modes have to be stored separately. More details on the numerical method as well as validation cases for the Navier-Stokes code can be found in the theses of Harris, ${ }^{23}$ von Terzi, ${ }^{24}$ and Mayer. ${ }^{25}$

\section{Boundary Conditions}

At the inflow, the conservative quantities $\rho, \rho u_{i}$, and $E_{t}$, obtained from the similarity solution of a compressible flat-plate boundary layer, are specified. The outflow is treated with a buffer domain technique ${ }^{26}$ to avoid reflections of disturbance waves. The buffer domain starts at $x_{3}$ and ends at $x_{L}$ with a length of $x_{L}-x_{3} \sim 0.5 \lambda_{x}$ (Fig. (1). At the free-stream boundary, all total flow quantities are separated into base flow and disturbance quantities. For the base-flow quantities, a homogeneous von Neumann condition is applied whereas for the disturbance quantities an exponential decay condition is employed that was derived for compressible flow using linear stability considerations. ${ }^{27}$ Periodicity is assumed in the spanwise direction. At the wall, the no-slip and no-penetration conditions are used except for the disturbance slot (see below). In addition, for the base flow, the wall temperature is set to the adiabatic wall temperature of the corresponding laminar flow, i.e. the initial condition, whereas temperature fluctuations are assumed to vanish.

\section{Disturbance Generation}

The flow is forced through the disturbance slot by prescribing a time-harmonic function for the fundamental spanwise Fourier mode of the $v$-velocity. During the start-up of the simulation, the forcing amplitude $A(\beta)$ 
is ramped up in time over one disturbance period. The velocity distribution $v_{p}$ over the blowing and suction slot has the shape of a dipole and it is represented by a fifth-order polynomial that is smooth everywhere including at the end points

$$
v\left(x_{p}, y=0, \beta, t\right)=A(\beta) v_{p}\left(x_{p}\right) \cos \left(-\omega t+\theta_{p}(\beta)\right) .
$$

$x_{p}$ is defined as

$$
x_{p}(x)=\frac{2 x-\left(x_{2}+x_{1}\right)}{x_{2}-x_{1}} \text { for }-1 \leq x_{p} \leq 1,
$$

and $v_{p}$ as

$$
v_{p}\left(x_{p}\right)=\left\{\begin{array}{l}
1.5^{4}\left(1+x_{p}\right)^{3}\left(3\left(1+x_{p}\right)^{2}-7\left(1+x_{p}\right)+4\right), x_{p} \leq 0 \\
-1.5^{4}\left(1-x_{p}\right)^{3}\left(3\left(1-x_{p}\right)^{2}-7\left(1-x_{p}\right)+4\right), x_{p}>0 .
\end{array}\right.
$$

The amplitude $A=0.003$ and, without any loss of generality, $\theta_{p}=0$ for the DNS described herein.

\section{E. Post-processing}

In order to obtain the spectral composition of the disturbance field from the DNS in a post-processing step, the physical time signal is Fourier transformed in time. The post-processing tool is based on the EAS3 tool kit from the Universität Stuttgart (http://en.wikipedia.org/wiki/EAS3). In EAS3, the Fourier transformations are given as follows

$$
\begin{aligned}
& \text { physical } \rightarrow \text { spectral: } \\
& \qquad \begin{aligned}
\widetilde{\phi}_{0} & \sim \frac{1}{n_{t}} \sum_{n=0}^{n_{t}-1} \phi_{n}, \\
\widetilde{\phi}_{m} & \sim \frac{1}{n_{t}} \sum_{n=0}^{n_{t}-1} 2 \phi_{n} \exp \left(\frac{-i 2 \pi m n}{n_{t}}\right),
\end{aligned}
\end{aligned}
$$

for $m=1, \ldots, M-1$ and $M=n_{t} / 2$. Here, $M$ contains the number of Fourier modes and $n_{t}$ the number of sample points of the physical signal. The output data of the tool is the absolute value $\left|\widetilde{\phi}_{m}\right|$ and the negative phase $-\arg \left(\widetilde{\phi}_{m}\right)$ of the complex number $\widetilde{\phi}_{m}$. The back transformation is defined as

spectral $\rightarrow$ physical:

$$
\phi_{n} \sim \operatorname{Re}\left[\sum_{m=0}^{M-1} \widetilde{\phi}_{m} \exp \left(\frac{i 2 \pi m n}{n_{t}}\right)\right],
$$

for $n=0, \ldots, n_{t}-1$.

\section{Simulation Setup for the PSE Approach}

In this section, the simulation setup of the PSE calculation is given. The computational domain differs from the DNS due to the different theoretical and numerical approaches. A short discussion on the numerical method, the boundary conditions and initial condition is also provided.

\section{A. Computational Setup}

The Reynolds number range and the disturbance frequency of the PSE calculations and of the DNS (CASE 3) are depicted in the stability diagram of Fig. 2 Two main PSE calculations have been performed, which vary in the starting position for the marching procedure. For one PSE calculation, from hereon abbreviated as PSE 1, the starting position is located close to the first neutral branch at $x_{0}^{P S E 1}=0.068 m\left(R_{x} \simeq 385\right)$ whereas, for the second PSE calculation, PSE 2 , the domain starts at $x_{0}^{P S E 2}=0.6 m\left(R_{x} \simeq 1144\right)$. The reasoning behind these choices is explained in section V[2 The domain height is chosen as $200 \times L^{*}$, with $L^{*}$

being the similarity boundary-layer length scale. The boundary layer is disturbed by prescribing non-parallel eigenfunctions obtained from a precursor non-parallel stability analysis (for a frequency of $f^{*}=6.36 \mathrm{kHz}$ 


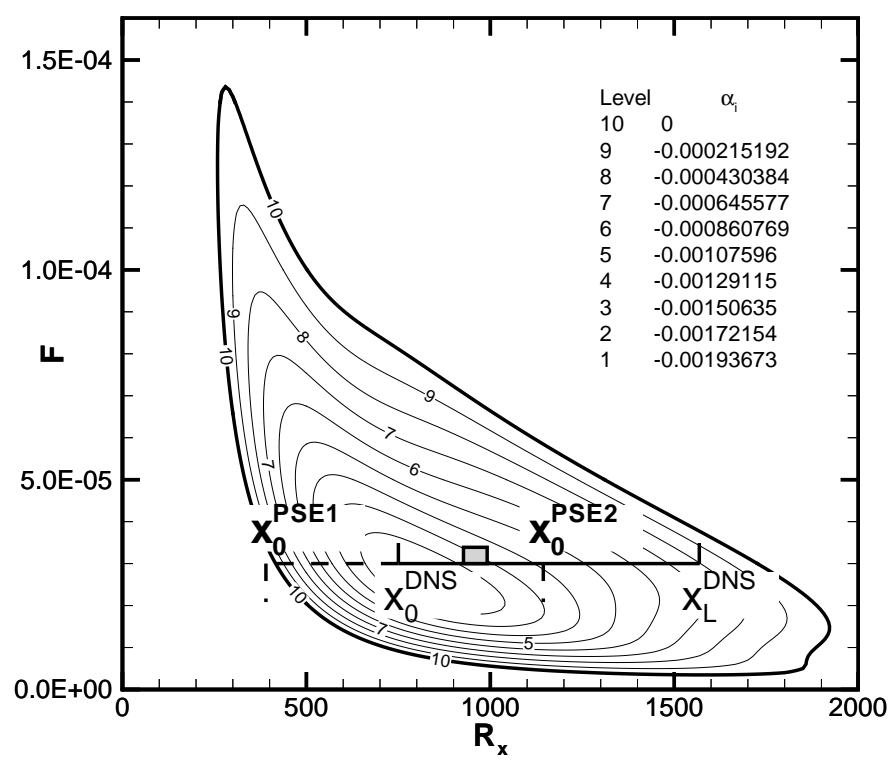

Figure 2. Stability diagram for $\beta^{*} \simeq 211.52 m^{-1}$ used to illustrate the computational domain for the PSE calculations. The box between $R_{x}=927$ and $R_{x}=993$ indicates the position of the blowing and suction slot in the DNS.

and a spanwise wave number of $\beta^{*} \simeq 211.52 \mathrm{~m}^{-1}$ ) at the inflow. The spanwise direction is assumed to be symmetric and is discretized using Fourier transformations. The number of Fourier modes for the oblique breakdown computations is selected to ensure an $\mathrm{O}\left(10^{-4}\right)$ decay in amplitude from the most energetic modes to the tail of the disturbance spectrum. The wall-normal direction is resolved by 171 points with clustering of grid points near the surface as well as the critical layer of the first-mode type waves.

\section{B. Numerical Method}

The governing linear and nonlinear PSEs are solved by a fourth-order central difference scheme in the wallnormal direction and by a first- or second-order one-sided difference scheme in the streamwise direction. The spanwise direction is assumed to be periodic and therefore transformed into spectral space using Fourier transforms. Furthermore, the spanwise discretization is pseudo-spectral, ${ }^{22}$ i.e. all nonlinear terms on the right-hand side of Eq. (12) are computed in physical space and then transformed back into spectral space. As for the DNS, the spanwise direction can be set to be symmetric in $u$-velocity, $v$-velocity, temperature, density, and pressure and antisymmetric in $w$-velocity reducing the computational domain to half a fundamental spanwise wave length $\lambda_{z}^{*}$. This approach is adopted for the present paper. Details on the discretization scheme can be found in Chang. ${ }^{28}$

\section{Initialization of PSE Calculations and Boundary Conditions}

To initialize the PSE analysis, mean-flow data for all streamwise positions and the disturbance information at the starting location of the streamwise marching procedure are required. A convenient way to initialize the PSE analysis is to prescribe eigenfunctions from LST. This approach, however, would result in a "transient" region where the PSE solution transitions from the LST eigenfunctions, satisfying parallel theory, at the inflow to the solution of the non-parallel parabolized stability equations. Hence, in the present work, eigenfunctions obtained from a precursor non-parallel stability analysis are used. The mean-flow data stems from a compressible similarity solution with adiabatic wall boundary condition. The differences between the similarity solution and the DNS mean flow were found to be negligible. At the wall, the no-slip condition is enforced and temperature fluctuations are set to zero while at the free stream, non-reflective boundary conditions are employed. 


\section{Results and Discussion}

The results section is split into three main parts. The first part gives a summary of the main findings for CASE 3 in the transitional regime, which are similar to CASE 2 in Mayer et al. ${ }^{11}$ Note that CASE 3 is symmetric in spanwise direction since, so far in the literature, oblique breakdown has always been initiated by two symmetric, oblique, first-mode type instability waves. Furthermore, the results of CASE 3 are complemented by discussing CASE 7, for which the symmetry restriction is removed. The second part of this section (section $\mathrm{V}[\mathrm{B}$ ) is focused on longer time averages for the turbulent regime and their comparison with shorter time averages from Mayer et al. ${ }^{12}$ The final part of the results section (section VC) provides a comparison of PSE results obtained from NASA's LASTRAC code to results from CASE 3 for the receptivity regime and the early nonlinear transition regime of oblique breakdown. This comparison provides additional validation of the DNS setup and the nonlinear PSE approach of LASTRAC.

\section{A. Removing the Symmetry Condition in the Spanwise Direction}

Before the influence of the symmetry condition on CASE 3 is evaluated using results from the new asymmetric simulation (CASE 7), some main findings for CASE 3 from the transitional regime are summarized here. As already mentioned in Mayer et al. ${ }^{11}$ and in von Terzi et al. ${ }^{14}$ the initial growth of the forced modes $[1, \pm 1]$ starts to deviate from linear growth when the higher-harmonic modes, as for example the stationary modes $[0, \pm 2]$ or modes $[1, \pm 3]$, reach comparable amplitude levels to modes $[1, \pm 1]$ (see Fig. [3 ). This event marks the end of the early nonlinear regime (at about $x^{*}=0.7 \mathrm{~m}$ ). Note that the notation $[h, k]$ is used to identify a particular wave according to its frequency $h$ and its spanwise wave number $k$. $h$ denotes multiples of the fundamental frequency and $k$ represents multiples of the smallest spanwise wave number.
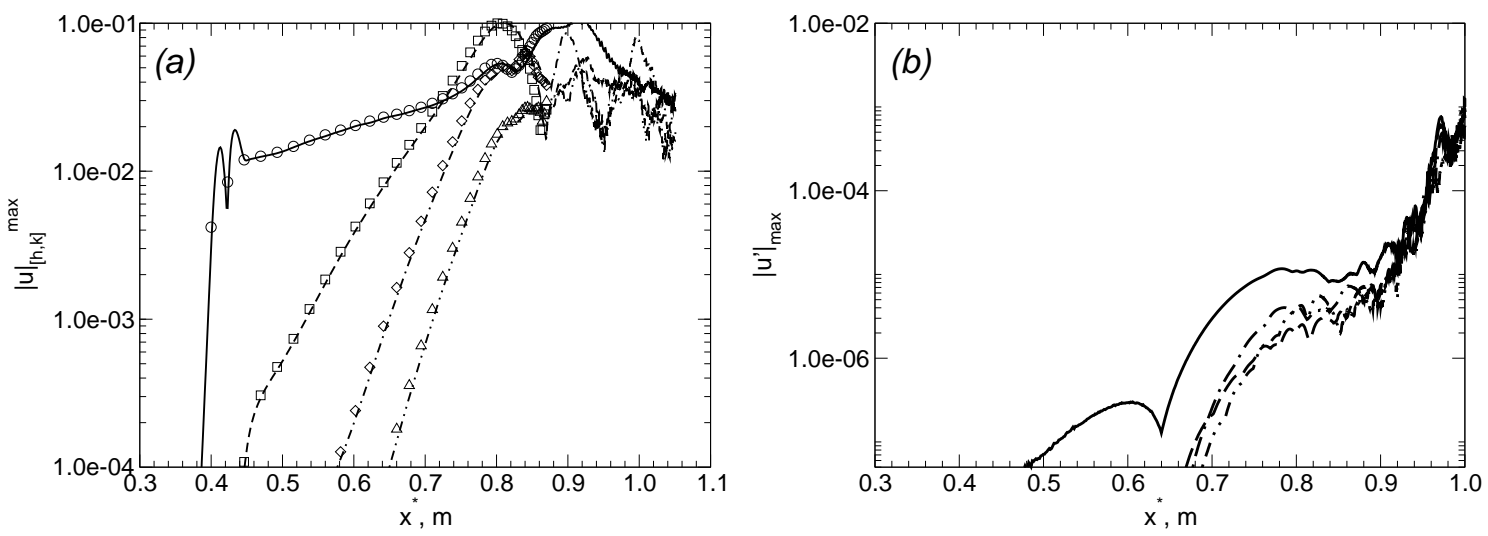

Figure 3. Streamwise amplitude development of mode shape maximum for modes with fundamental frequency (a) and subharmonic frequency from CASE 3 (b): For (a): (o, - ) $[1, \pm 1],(\square,--)[1, \pm 3],(\diamond,-.-)[1, \pm 5],(\Delta,-.$. $[1, \pm 7])$; For (b): (-) $[0.5,0],(--)[0.5, \pm 1],(-.-)[0.5, \pm 2],(-.).[0.5, \pm 3]$. Results in (a) are from CASE 1 and 2 in Mayer et al. ${ }^{11}$ and in (b) from CASE 3.

At about $x^{*}=0.8 m$, nonlinear saturation of the $u$-velocity disturbance amplitude sets in (Fig. 31). Modes that are not directly generated by nonlinear wave interactions from the initial forced modes $[1, \pm 1]$, as for example modes with subharmonic frequency in Fig. [3 $\mathrm{b}$, also experience amplitude growth and saturation at about $x^{*}=0.8 \mathrm{~m}$. These modes are initialized by round-off errors due to the limited machine precision. One major difference between Fig. 3 $\mathrm{a}$ and Fig. 3b is the sudden increase in the streamwise growth rate for all modes with subharmonic frequency at about $x^{*}=0.9 \mathrm{~m}$. This sudden increase in amplification seems to be linked to the breakup of large longitudinal structures into small-scales as depicted in Fig. 目 (between about $x^{*}=0.84 m$ and $x^{*}=0.9 m$ ). This figure shows instantaneous three-dimensional isosurfaces obtained from the $\mathrm{Q}$-criterion ${ }^{29}(\mathrm{Q}=15000)$. $\mathrm{Q}$ is related to the second invariant of the velocity gradient tensor. Positive values of $\mathrm{Q}$ reveal flow regions where rotation dominates the strain.

The flow structures in Fig. 4 are symmetric with respect to the centerplane of the domain. This is to be expected since these figures are obtained from CASE 3, where symmetry is enforced. If the symmetry condition is not enforced as for CASE 7, the picture does not change as demonstrated by Fig. 5 . This figure illustrates contours of streamwise velocity $u$ of the first higher Fourier mode $(k=1)$ in spanwise direction from CASE 7 for the sine and cosine modes (Eq. 19), respectively. The minimum and maximum of the 


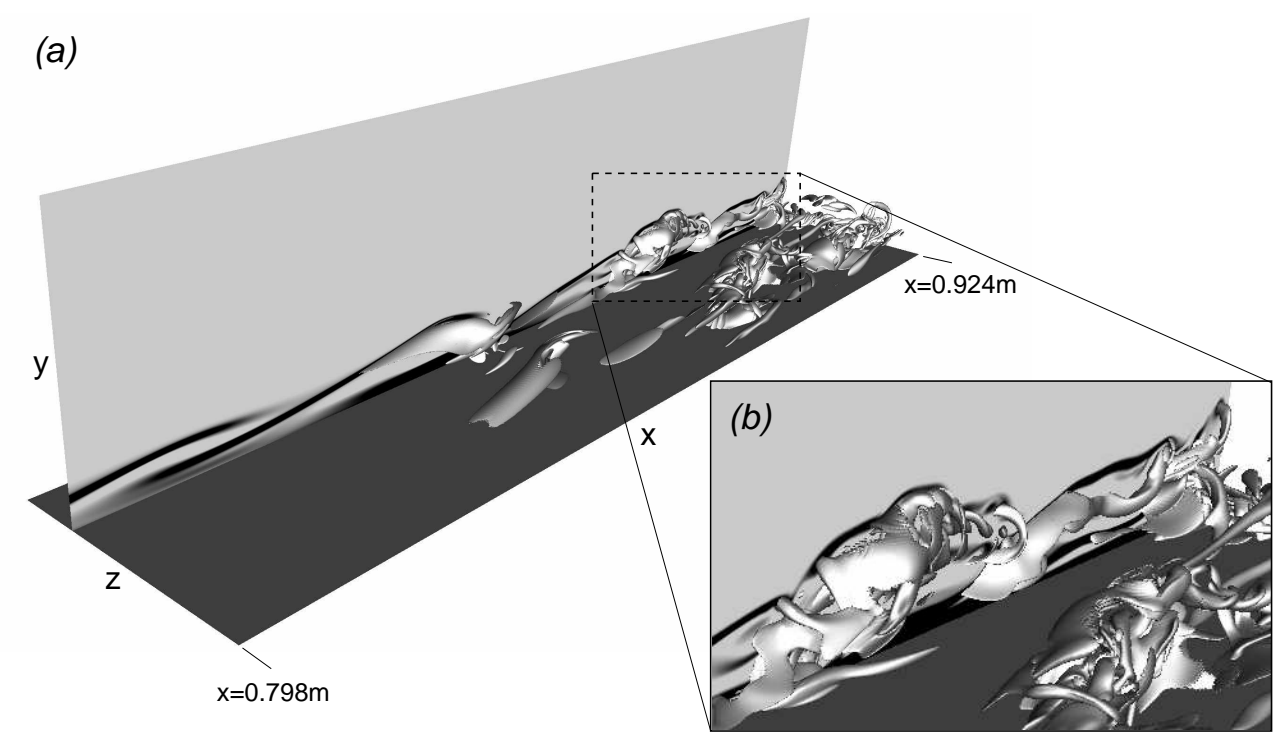

Figure 4. Instantaneous flow structures identified by the Q-criterion for $Q=15000$ (CASE 3) between $x^{*}=0.798 m$ and $x^{*}=0.924 m$. Also shown are contours of spanwise vorticity at $z^{*} \simeq-0.0087 \mathrm{~m}$. (a) Entire three-dimensional view, (b) close-up of the breakdown region confirming that the Q-criterion predicts similar structures as illustrated by the spanwise vorticity; $M=3.0, T_{\infty}^{*}=103.6 \mathrm{~K}$, flat plate.

contour levels in Figs. 5 and $\mathrm{b}$ are different in order to emphasize the flow structures. The influence of asymmetric modes on oblique breakdown initiated by two oblique waves with exactly the same amplitude and phase is limited since these modes are only generated by the round-off error of the calculation. For CASE 3, the streamwise position of the final breakup into small-scale structures denotes the location where all modes with frequency unequal to integer multiples of the forcing frequency are strongly amplified (at about $x^{*}=0.9 \mathrm{~m}$ in Fig. $3 \mathrm{k}$ ). For CASE 7 , a similar behavior can be observed. At the same streamwise position (where the breakup into small-scale structures occurs) the asymmetric modes also start to become amplified as illustrated by Fig. [5 $\mathrm{k}$. The amplitude values of the streamwise velocity for the sine mode in Fig. 5 provide a measure of the magnitude of asymmetry in CASE 7 . For the $u$-velocity, this mode is set to zero in CASE 3. Since the contour levels for the sine mode in Fig. 5 a are more than 10 orders of magnitude smaller than the contour levels for the cosine mode, CASE 7 remains essentially symmetric even after the breakup into small-scale structures. This is true over the entire domain length of CASE 3. It is however evident that the asymmetric modes are strongly amplified in the downstream direction and will eventually reach high amplitude levels.

\section{B. Longer Time-Averaging}

The early turbulent regime for oblique breakdown (CASE 3) is discussed in detail in Mayer et al. ${ }^{12}$ It is shown how the skin friction coefficient increased in the streamwise direction during transition until a peak was reached. Downstream of the peak, a loss of periodicity in the time signals for the investigated setup occurred and the skin-friction approached correlations and comparable data for turbulent boundary layers in the literature. Furthermore, a logarithmic region in the van Driest transformed mean streamwise velocity profile was detected and power spectra of the velocity components matched well-known theoretical scaling laws.

In Mayer et al., ${ }^{12}$ turbulent quantities are calculated from data, which are time-averaged according to

$$
\bar{\phi}=\frac{1}{\lambda_{z}} \frac{1}{\Delta t} \int_{0}^{\lambda_{z}} \int_{t_{0}}^{t_{0}+\Delta t} \phi(t, z) d t d z
$$

Moreover, fluctuations about the mean of a Reynolds-averaged quantity $\bar{\phi}$ are denoted by $\phi^{\prime}$ and fluctuations about the mean of a Favre-averaged quantity $\overline{\rho \phi} / \bar{\rho}$ are denoted by $\phi^{\prime \prime}$. The interval length $\Delta t$ is, however, only 12 forcing periods $T_{\text {forcing }}$ in Mayer et al. ${ }^{12}$ Whether this interval length is sufficiently long, is discussed in this section. Fig. [6 demonstrates the skin-friction coefficient as a function of the interval length for the 

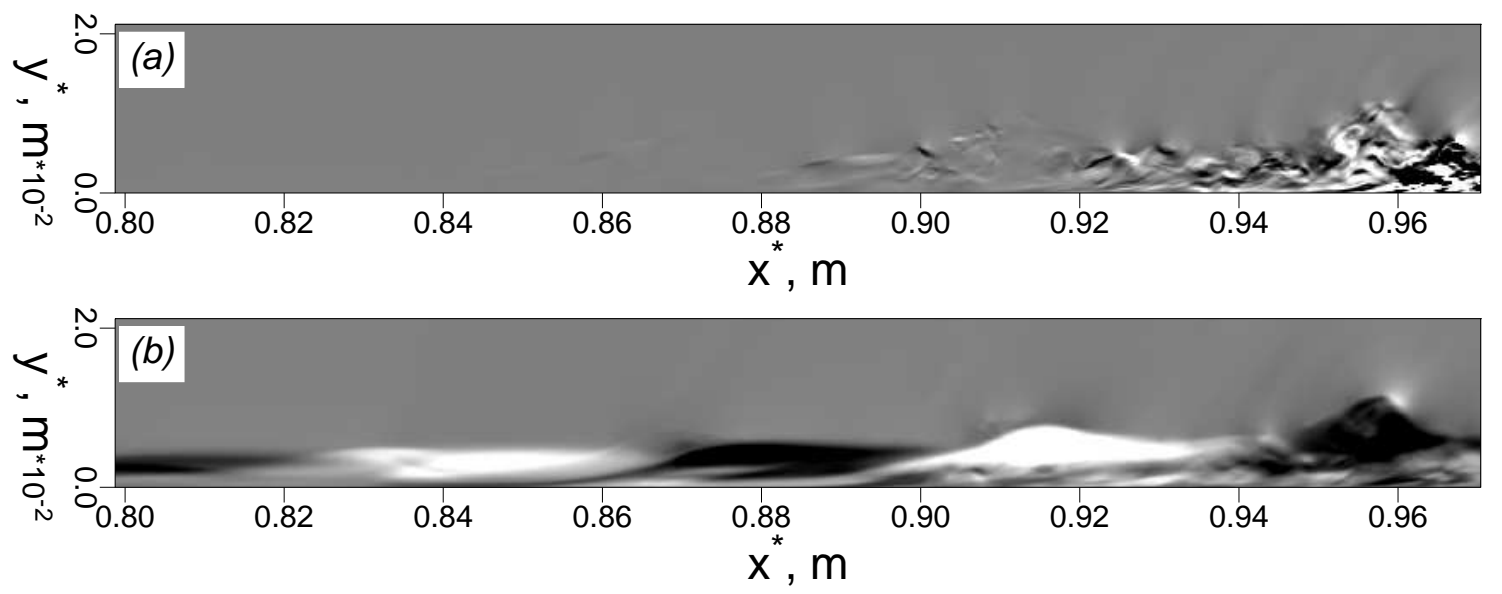

Figure 5. Contours of instantaneous streamwise velocity $u$ obtained from CASE 7 for the first higher Fourier mode in spanwise direction: (a) sine mode, contour levels from $-1.0 \mathbf{E}-12$ to $1.0 \mathbf{E}-12$, (b) cosine mode, contour levels from -0.1 to 0.1 .

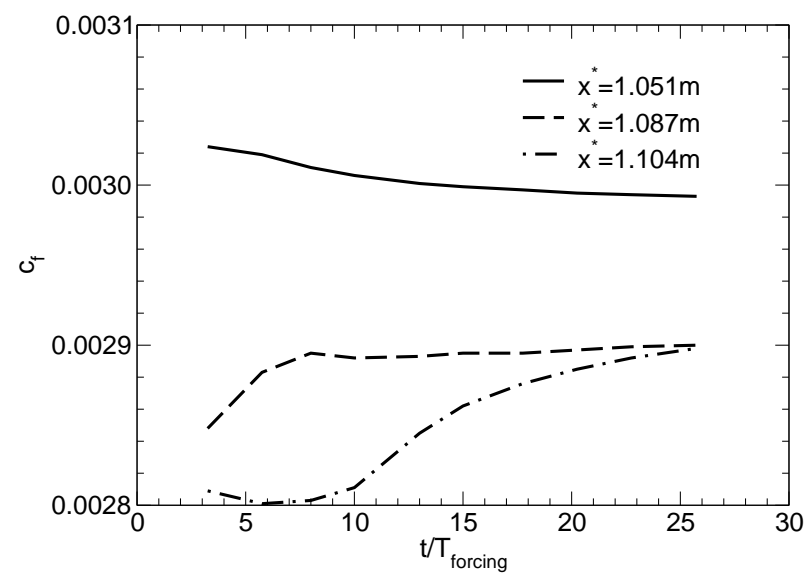

Figure 6. Skin-friction coefficient for CASE 3 as a function of interval length for time-averaging indicated by the number of forcing periods $T_{\text {forcing }}$ at three different streamwise positions.

time average at three different streamwise positions. These positions are downstream of the skin-friction peak, within the early turbulent region, where the flow starts to lose its temporal periodicity with respect to the forcing frequency. For the first two positions, the skin-friction coefficient does not change significantly when the interval length for the time average is increased. This is most likely due to the fact that the flow field still exhibits some temporal periodicity and a negligible low-frequency disturbance content. Hence, a time interval of 12 forcing periods seems to be sufficient for a good time average. At the last position $\left(x^{*}=1.104 m\right)$, however, a longer time average is required (even longer than $\left.\Delta t / T_{\text {forcing }}=25.75\right)$. Time signals from this position are shown in Mayer et al. ${ }^{12}$ and they demonstrate that the flow field has lost its temporal periodicity at this location. A similar conclusion can be drawn from Fig. 7 which illustrates the streamwise distribution of selected mean-flow properties from CASE 3 for two different time averages. The curves with 12 forcing periods as time-average interval are very close to the curves with 25.75 forcing periods.

As can be seen in Figs. [6 and 7 the increase in interval length for the time average does not strongly alter the mean values for CASE 3. However, the impact on fluctuation quantities, as for example the r.m.s. values, might be more pronounced. Therefore, Fig. 8 compares the r.m.s. values of the streamwise velocity component and the Reynolds shear stress $\overline{u^{\prime \prime} v^{\prime \prime}}$ obtained from data with $\Delta t / T_{\text {forcing }}=12$ to the data with $\Delta t / T_{\text {forcing }}=25.75$ at $x^{*}=1.087 \mathrm{~m}$. In addition, profiles published in the literature from the temporal DNS 

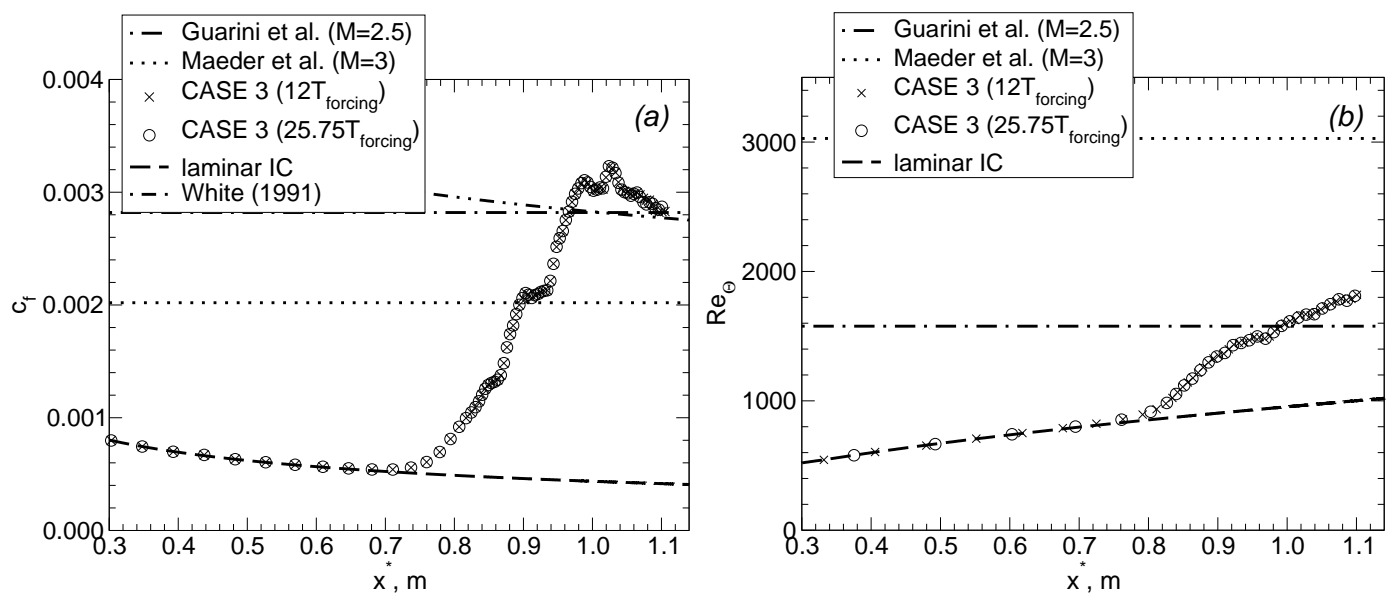

Figure 7. Streamwise development of selected mean-flow properties from CASE 3 in comparison to different values published in the literature for turbulent supersonic flow ${ }^{30,31}$ and theoretical models: ${ }^{32}$ (a) skin-friction coefficient $c_{f}$, (b) Reynolds number based on momentum thickness $\Theta$.

by Guarini et al..$^{30}$ of a compressible turbulent boundary layer at Mach 2.5 are also included in this figure. The profiles from the different time averages are in surprisingly good agreement leading to the conclusion that even for the fluctuation quantities, a time interval of $\Delta t / T_{\text {forcing }}=12$ for the average is satisfactory up to $x^{*}=1.087 m$.
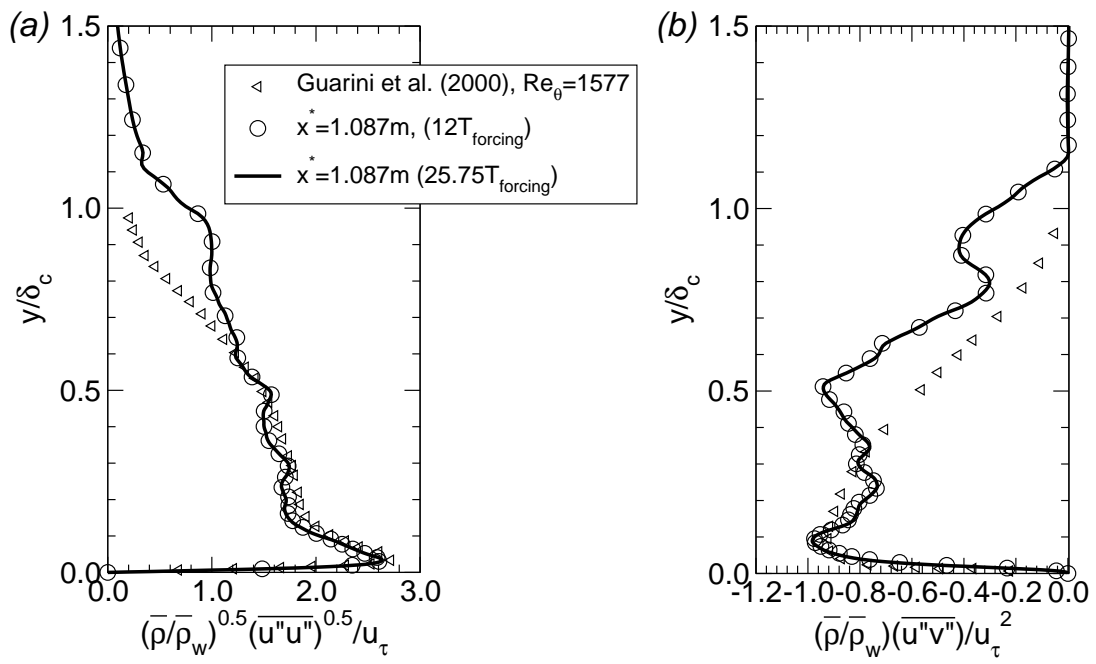

Figure 8. Wall-normal distribution of r.m.s. values in streamwise direction (a) and Reynolds shear stress $\overline{u^{\prime \prime} v^{\prime \prime}}$ (b) at $x^{*}=1.087 m$ for CASE 3 .

\section{PSE Comparison}

As a short summary, the first two sections (section VA and V $\mathrm{B}$ ) confirmed that CASE 3 from Mayer et al. ${ }^{12}$ is a representative case for oblique breakdown at Mach 3. The symmetry condition is applicable for the computational setup of CASE 3 and the data was sufficiently time-averaged. In this section, we employ NASA's LASTRAC code in order to study its nonlinear PSE capabilities and to evaluate the simulation setup of CASE 3 in the receptivity regime and early nonlinear regime.

\section{Receptivity Study}

In order to investigate the receptivity behavior of a laminar Mach 3 boundary layer with adiabatic wall, LASTRAC's receptivity module is applied. This module can be used for making integrated predictions of 
receptivity and subsequent evolution of instability waves for certain simple classes of receptivity mechanisms, including the receptivity to unsteady blowing and suction at the surface (as in CASE 3). Before presenting any results for the instability wave perturbations excited by the unsteady blowing and suction slot from Eq. (20), we intend to explain the receptivity behavior of a supersonic boundary layer to this form of forcing in general. As described in the context of the DNS results, a localized blowing and suction slot excites the first mode-type instability wave at the frequency (and spanwise wave number) of excitation as well as other disturbances that are stable and, hence, do not influence the disturbance dynamics sufficiently far downstream. We confine our attention to the instability wave portion of the boundary layer response and examine how well the theoretical models (and, in particular, the model based on adjoint $\mathrm{PSE}^{20}$ ) can predict the first-mode type perturbations in this specific case. The theoretical prediction for the instability wave perturbations due to the surface actuator can be expressed in the form of Eq. (11), where

$$
\epsilon=\frac{1}{\sqrt{2 \pi}} \int_{x_{0}}^{x} \Lambda(\xi, \beta) \widetilde{F}(\xi, \beta) \exp \left(-i \theta_{r}(\xi, \beta)\right) d \xi
$$

Here, the efficiency function $\Lambda$ represents the instability wave portion of the Green's function associated with the boundary layer response to this type of wall forcing, i.e., the effective initial amplitude of the instability wave due to a point source actuation. It characterizes the intrinsic receptivity characteristics of the local boundary layer to a specific type of forcing and is independent of the spatial distribution of the source. The dependence on the source geometry is reflected via the convolution of the efficiency function $\Lambda$ with the spanwise Fourier transfrom of the unsteady blowing and suction velocity $v_{p}$ from Eq. (20)

$$
\widetilde{F}(x, \beta)=\frac{1}{2} A v_{p}(x) .
$$

The quantity $\theta_{r}$ in Eq. (26) denotes the phase of the velocity perturbation

$$
\theta_{r}(x, \beta)=\int_{x_{0}}^{x} \alpha_{r}(\xi, \beta) d \xi
$$
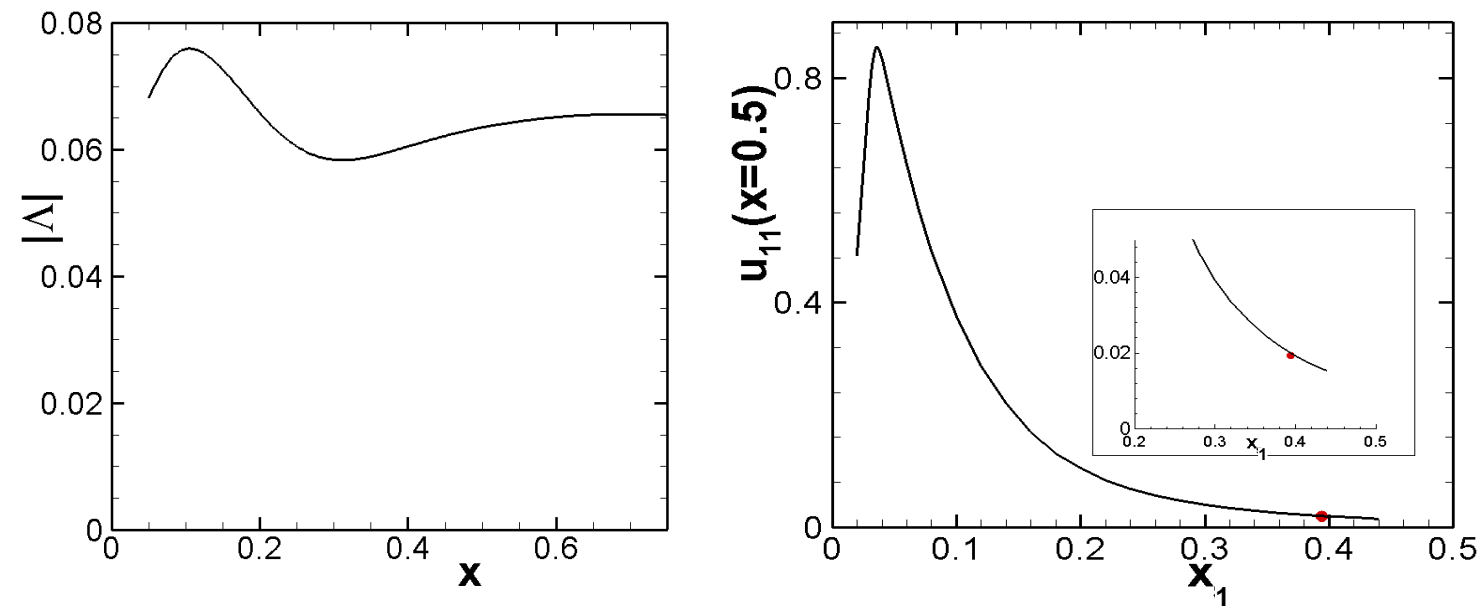

Figure 9. Receptivity behavior of a laminar Mach 3 boundary layer with adiabatic wall due to blowing and suction according to Eq. (20) applied in CASE 3: (a) efficiency function $\Lambda$ from PSE as a function of streamwise position $\mathrm{x}$, and (b) predicted amplitude value for modes [1, \pm 1 ] from PSE (solid line) and from DNS (dot) at position $x^{*}=0.5 \mathrm{~m}$ as a function of starting position of disturbance slot $x_{1}$. Note that the inset in (b) shows the combined amplitudes of modes $[ \pm 1, \pm 1]$.

Fig. 9 illustrates the magnitude of the efficiency function $\Lambda$, plotted as a function of the streamwise source location $x$. The choice of normalization of the efficiency function is the same as in Chang and Choudhari. ${ }^{20}$ It can be seen in Fig. 9, that the intrinsic efficiency values vary weakly with the source location, with less than $33 \%$ variation over a broad range of actuator locations. Thus, the dominant effect of source location on the amplitude of modes $[1, \pm 1]$ is due to the progressive reduction in the linear amplification potential 
associated with source locations downstream of the first neutral branch. This effect is reflected in the strong reduction in the amplitude of modes $[1, \pm 1]$ at $x^{*}=0.5 \mathrm{~m}$ as the starting location for the blowing and suction slot is varied along the streamwise direction in Fig. 9b. Note that, for the results plotted in this figure, the measurement location is sufficiently downstream of the source, but still within a predominantly linear region in terms of the fundamental mode evolution. The amplitude of the instability wave extracted from the DNS computation (shown as a dot in Fig. 9p) is within $3 \%$ of the value predicted by the theory. This small discrepancy could be even further narrowed by accounting for the cumulative effects of disturbance nonlinearity between the disturbance slot and the measurement location, but was deemed unnecessary.

\section{Finite Amplitude Forcing}

The early nonlinear regime of oblique breakdown at Mach 3 is studied by performing PSE calculations with two different simulation setups. As already mentioned in section IV A the first setup starts at the first neutral branch (PSE 1) while the second PSE calculation starts at $x^{*}=0.6 m$ (PSE 2). For PSE 1, the initial disturbance amplitudes of modes $[1, \pm 1]$ at the neutral branch are scaled in order to match the absolute amplitude values from the DNS farther downstream for this mode, while for PSE2, the initial amplitudes of the first 6 dominant modes $([1, \pm 1],[0, \pm 2]$, and $[1, \pm 3])$ are scaled to the corresponding values from the DNS at $x^{*}=0.6 \mathrm{~m}$. The reasoning for the setup PSE 1 is to examine whether the DNS results (CASE 3) can be
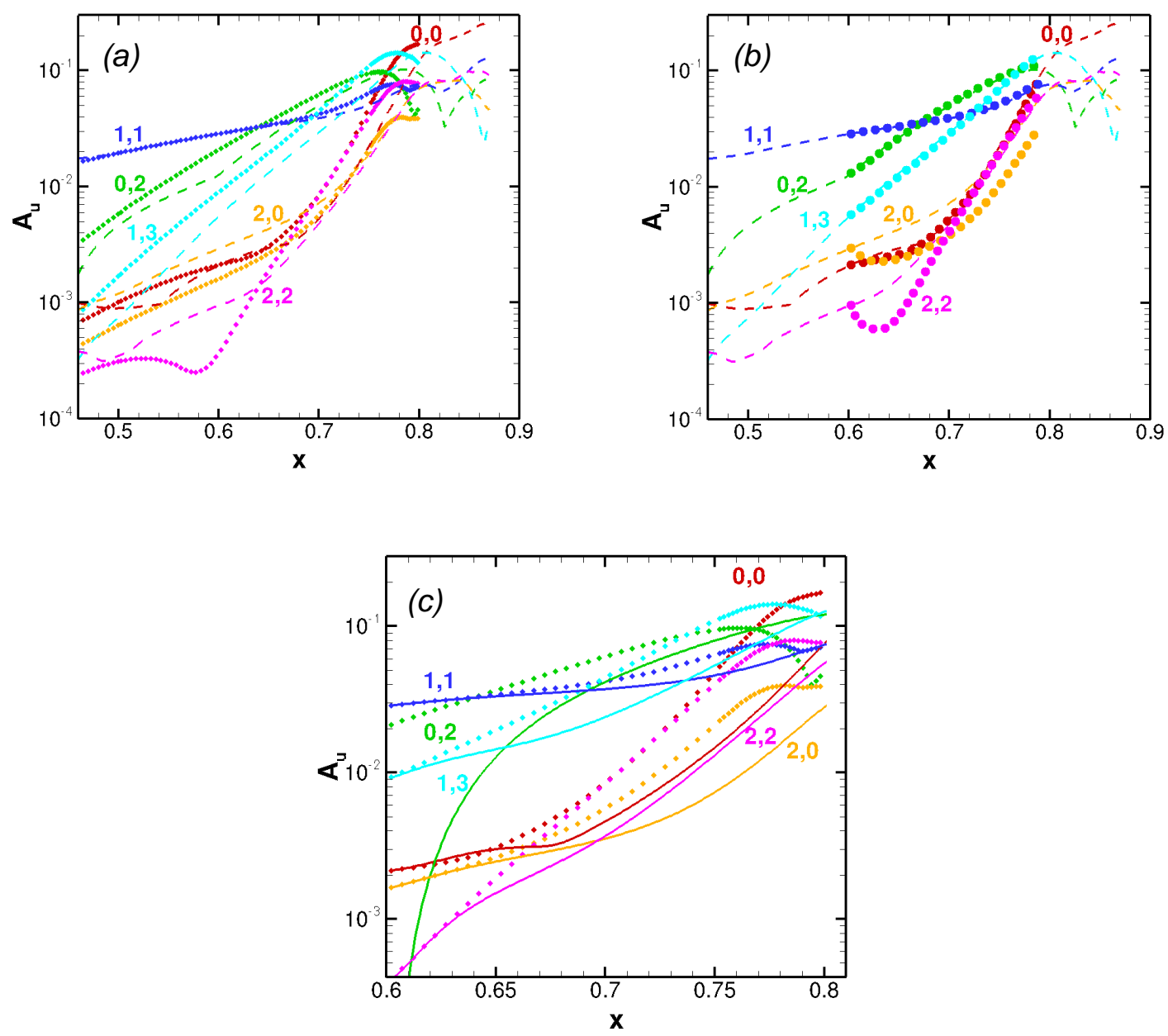

Figure 10. Streamwise amplitude development of the wall-normal maximum (mode shape maximum) for selected modes calculated from physical data according to the Fourier transforms given in Eqs. (15) and (23): (a) (symbols) PSE 1, (dashed lines) DNS, (b) (symbols) PSE 2, (dashed lines) DNS. (c) Setup from PSE 1, but with (solid lines) and without (symbols) the suppression of modes $[0, \pm 2]$. 
completely recovered by a PSE calculation starting with a very low disturbance amplitude at the first neutral branch, which would be the case during natural transition (i.e. without an artificial disturbance source at the plate surface as employed in the DNS). As shown in Fig. 10k, which illustrates the streamwise amplitude development of the wall-normal maximum (mode shape maximum) for selected modes, the DNS cannot be completely recovered resulting in a discrepancy between the nonlinear generated modes from the DNS and the nonlinear PSE analysis. This difference is mainly caused by nonlinear effects during the receptivity process in the DNS since the forcing amplitude of the DNS is already quite large $(\mathrm{A}=0.3 \%)$. If in a second PSE analysis the setup is changed to PSE 2 so that the amplitudes of not just the fundamental, but also the most dominant nonlinearly excited modes are matched with the DNS, then the agreement between the data from PSE and the data from DNS improves significantly as demonstrated in Fig. 10b.

We also examined the effect of suppressing modes $[0, \pm 2]$ to disrupt the energy cascade from modes $[1, \pm 1]$ to their higher-harmonic modes associated with oblique breakdown. Mode $[0, \pm 2]$ was canceled out by a modest stationary forcing at $x^{*}=0.6 \mathrm{~m}$ in a new PSE calculation for setup PSE 1 . Fig. 10p shows the results corresponding to this simulation. Symbols refer to the PSE results from Fig. 10k, where modes $[0, \pm 2]$ are not suppressed and solid lines represent the new simulation. Clearly, the influence of nonlinear wave interactions is weakend resulting in a delay of transition. While the delay in transition onset location is approximately $5 \%$ increased, it may be less in a natural disturbance environment.
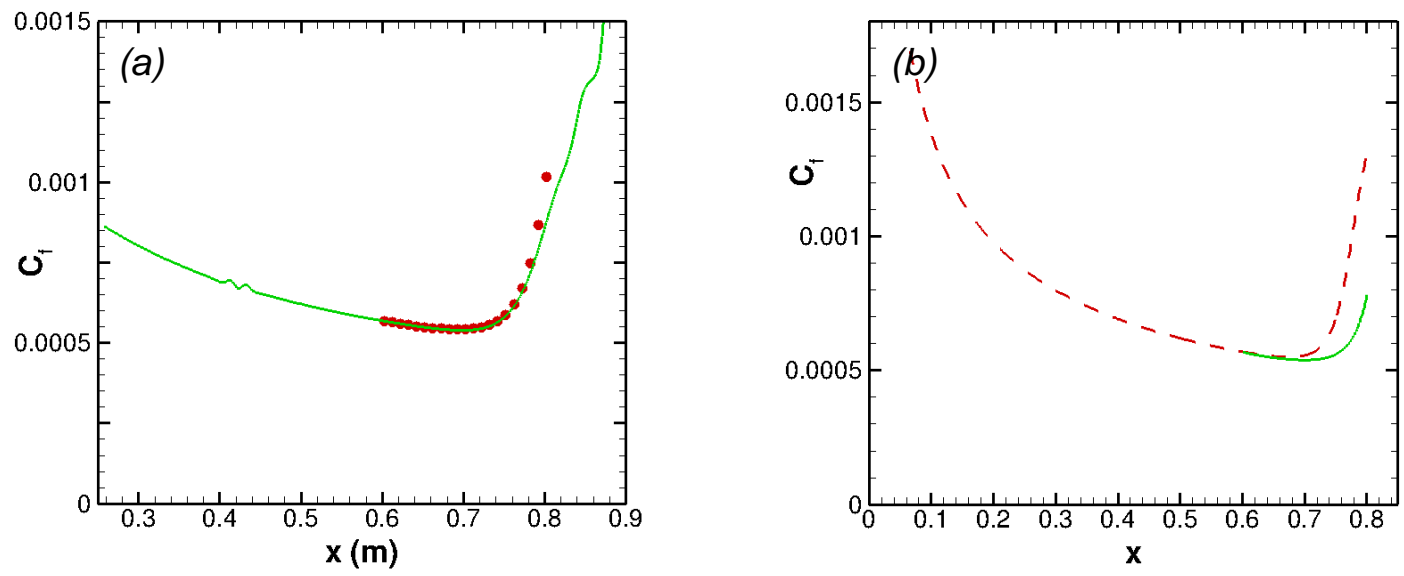

Figure 11. Streamwise development of skin friction coefficient: (a) (solid line) DNS, (symbols) PSE, (b) PSE with (solid line) and without (dashed line) suppression of modes $[0, \pm 2]$.

The previously mentioned findings are confirmed by the streamwise development of the skin friction coefficient in Fig. 11] The comparison between DNS and PSE results (PSE 2) in Fig. 117 leads to an excellent agreement between both methods indicating that nonlinear PSE is able to predict the transition onset correctly. In fact, the agreement extends up to the location, where the skin friction coefficient is nearly twice the value of the underlying laminar skin friction. This suggests that (i) the PSE results could, perhaps, be bridged with a turbulence model to provide integrated predictions of transition and turbulence ${ }^{33}$ and that such PSE results could be used, as a cost effective means, to initiate simulations of fully turbulent boundary layers. For the case with suppressed modes $[0, \pm 2]$, the transition onset is indeed moved downstream as depicted in Fig. 111b.

\section{Conclusions}

Transition to turbulence via oblique breakdown in a Mach 3 boundary layer was investigated using DNS and PSE. Our previous studies of the same case focused on the detailed documentation of the different transition stages and demonstrated that oblique breakdown can lead to a fully developed turbulent boundary layer. In these studies, however, the flow was assumed to be symmetric in the spanwise direction. A new DNS was performed where the symmetry condition was removed. This simulation demonstrated that oblique breakdown, initialized by two oblique instability waves with exactly the same amplitude level, loses its symmetry late in the turbulent stage for a low-noise environment. Hence, for the streamwise extent of 
the computational domain in this study, the symmetry condition is justified. Furthermore, new data from a longer time average of the original symmetric simulation of oblique breakdown (CASE 3) was discussed. These data confirm that a converged time average is reached. The final part of the paper focused on a comparison of PSE results obtained from NASA's LASTRAC code to the DNS results all the way from the disturbance generation to the initial part of the breakdown process. Good agreement was found for all different transition stages, including receptivity, linear, and nonlinear disturbance evolution, and the initial part of the breakdown process until the mean wall shear had nearly doubled with respect to its laminar value. Thus, the present paper corroborates that our oblique breakdown case for Mach 3 constitutes a generic reference case that can be successfully used to validate different engineering tools, as for example the PSE approach, across the different transition stages. Furthermore, the PSE approach combined with turbulence models for transitional flow might be a sophisticated solution for modeling the entire transition process of a supersonic boundary layer.

\section{Acknowledgments}

This work was partially funded by the Air Force Office for Scientific Research under grant FA9550-08-10211 with Dr. John Schmisseur serving as program manager. The computer hours and the technical support provided by NASA Ames are greatly acknowledged.

\section{References}

${ }^{1}$ Berry, S. A., Hamilton, H. H., and Wurster, K. E., "Effect of Computational Method on Discrete Roughness Correlations for Shuttle Orbiter," Journal of Spacecraft and Rockets, Vol. 43, No. 4, 2006, pp. 842-852.

${ }^{2}$ Reshotko, E., "Is $R e_{\theta} / M_{e}$ a Meaningful Transition Criterion?" AIAA-2007-0943, 2007.

${ }^{3}$ Mack, L. M., "Boundary-Layer Stability Theory," Internal Document 900-277, Jet Propulsion Laboratory, Pasadena, California, 1969.

${ }^{4}$ Fedorov, A. V., "Receptivity of a high-speed boundary layer to acoustic disturbances," J. Fluid Mech., Vol. 491, 2003, pp. 101-129.

${ }^{5}$ Tumin, A., "Three-Dimensional Spatial Normal Modes in Compressible Boundary Layers," J. Fluid Mech., Vol. 586, 2007, pp. 295-322.

${ }^{6}$ Fasel, H., Thumm, A., and Bestek, H., "Direct Numerical Simulation of Transition in Supersonic Boundary Layer: Oblique Breakdown," Transitional and Turbulent Compressible Flows, edited by L. D. Kral and T. A. Zang, No. 151 in FED, ASME, 1993, pp. 77-92.

${ }^{7}$ Kosinov, A. D., Semionov, N. V., Shevelkov, S. G., and Zinin, O. I., "Experiments on the Nonlinear Instability of Supersonic Boundary Layers," Nonlinear Instability of Nonparallel Flows, edited by D. T. Valentine, S. P. Lin, and W. R. C. Philips, Springer, 1994, pp. 196-205.

${ }^{8}$ Corke, T. C., Cavalieri, D. A., and Matlis, E., "Boundary-Layer Instability on Sharp Cone at Mach 3.5 with Controlled Input," AIAA J., Vol. 40, No. 5, 2002, pp. 1015-1018.

${ }^{9}$ Mayer, C. S. J., Wernz, S., and Fasel, H. F., "Investigation of Oblique Breakdown in a Supersonic Boundary Layer at Mach 2 Using DNS," AIAA-2007-0949, 2007.

${ }^{10}$ Mayer, C. S. J. and Fasel, H. F., "Investigation of Asymmetric Subharmonic Resonance in a Supersonic Boundary Layer at Mach 2 Using DNS," AIAA-2008-0591, 2008.

${ }^{11}$ Mayer, C. S. J., von Terzi, D. A., and Fasel, H. F., "DNS of Complete Transition to Turbulence Via Oblique Breakdown at Mach 3," AIAA-2008-4398, 2008.

${ }^{12}$ Mayer, C. S. J., von Terzi, D. A., and Fasel, H. F., "DNS of Complete Transition to Turbulence Via Oblique Breakdown at Mach 3: Part. II," AIAA-2009-3558, 2009.

${ }^{13}$ Mayer, C. S. J., Laible, A. C., and Fasel, H. F., "Numerical Investigation of Transition initiated by a Wave Packet on a Cone at Mach 3.5," AIAA-2009-3809, 2009.

14 von Terzi, D. A., Mayer, C. S. J., and Fasel, H. F., "The Late Nonlinear Stage of Oblique Breakdown to Turbulence in a Supersonic Boundary Layer," Seventh Symposium on Laminar-Turbulent Transition, edited by P. Schlatter and D. S. Henningson, Springer, 2009, pp. 415-420.

${ }^{15}$ Kosinov, A. D., Semionov, N. V., and Shevelkov, S. G., "Investigation of Supersonic Boundary Layer Stability and Transition Using Controlled Disturbances," Methods of Aerophysical Research, edited by A. M. Kharitonov, Vol. 2, 1994, pp. $159-166$.

${ }^{16}$ Ermolaev, Y. G., Kosinov, A. D., and Semionov, N. V., "Experimental Investigation of Laminar-Turbulent Transition Process in Supersonic Boundary Layer Using Controlled Disturbances," Nonlinear Instability and Transition in Three-Dimensional Boundary Layers, edited by P. W. Duck and P. Hall, Kluwer Academic Publishers, 1996, pp. 17-26.

${ }^{17}$ Kosinov, A. D., Maslov, A. A., and Semionov, N. V., "An Experimental Study of Generation of Unstable Disturbances on the Leading Edge of a Plate at M=2," J. Appl. Mech. Tech. Phys., Vol. 38, No. 1, 1997, pp. 45-50.

${ }^{18}$ Jiang, L., Choudhari, M., Chang, C.-L., and Liu, C., "Numerical Simulations of Laminar-Turbulent Transition in Supersonic Boundary Layer," AIAA-2006-3224, 2006. 
${ }^{19}$ Chang, C. L., "The Langley Stability and Transition Analysis Code (LASTRAC): LST, Linear \& Nonlinear PSE for 2-D, Axisymmetric, and Infinite Swept Wing Boundary Layers," AIAA-2003-0974, 2003.

${ }^{20}$ Chang, C. L. and Choudari, M., "Boundary-Layer Receptivity and Integrated Transition Prediction," AIAA-2005-0526, 2005.

${ }^{21}$ Graziosi, P. and Brown, G. L., "Experiments on Stability and Transition at Mach 3," J. Fluid Mech., Vol. 472, 2002, pp. $83-124$.

${ }^{22}$ Canuto, C., Hussaini, M., Quateroni, A., and Zang, T., Spectral Methods in Fluid Dynamics, Springer, 1988.

${ }^{23}$ Harris, P. J., Numerical Investigation of Transitional Compressible Plane Wakes, Ph.D. thesis, The University of Arizona, 1997.

${ }^{24}$ von Terzi, D. A., Numerical Investigation of Transitional and Turbulent Backward-Facing Step Flows, Ph.D. thesis, The University of Arizona, 2004.

${ }^{25}$ Mayer, C. S. J., Numerical Investigation of the Nonlinear Transition Regime in Supersonic Boundary Layers, Ph.D. thesis, The University of Arizona, 2009.

${ }^{26}$ Meitz, H. and Fasel, H. F., "A Compact-Difference Scheme for the Navier-Stokes Equations in Vorticity-Velocity Formulation," J. Comp. Phys., Vol. 157, 2000, pp. 371-403.

${ }^{27}$ Thumm, A., Numerische Untersuchungen zum laminar-turbulenten Strömungsumschlag in transsonischen Grenzschichtströmungen, Ph.D. thesis, Universität Stuttgart, 1991.

${ }^{28}$ Chang, C. L., "The Langley Stability and Transition Analysis Codes (LASTRAC) User Manual," Tech. Rep. TM, NASA, 2002.

${ }^{29}$ Hunt, J. C. R., Wray, A. A., and Moin, P., "Eddies, Streams, and Convergence Zones in Turbulent Flows," Proceedings of the 1988 Summer Conference, Stanford University, 1988.

${ }^{30}$ Guarini, S. E., Moser, R. D., Shariff, K., and Wray, A., "Direct Numerical Simulation of a Supersonic Turbulent Boundary Layer at Mach 2.5," J. Fluid Mech., Vol. 414, 2000, pp. 1-33.

${ }^{31}$ Maeder, T., Adams, N. A., and Kleiser, L., "Direct simulations of turbulent supersonic boundary layers by an extended temporal approach," J. Fluid Mech., Vol. 429, 2001, pp. 187-216.

${ }^{32}$ White, F. M., Viscous Fluid Flow, McGraw-Hill, 1991.

${ }^{33}$ Rubinstein, R. and Choudhari, M., "Statistical Prediction of Laminar-Turbulent Transition," Tech. Rep. CR-2000-210638, NASA, 2000. 